\title{
Comparison of fuzzy Adomian decomposition method with fuzzy VIM for solving fuzzy heat-like and wave-like equations with variable coefficients
}

\author{
Mawia Osman ${ }^{1} \mathbb{D}$, Zengtai Gong ${ }^{1 *}$ and Altyeb Mohammed Mustafa, ${ }^{1,2}$
}

\section{"Correspondence:}

zt-gong@163.com

${ }^{1}$ College of Mathematics and

Statistics, Northwest Normal

University, Lanzhou, Gansu,

P.R. China

Full list of author information is

available at the end of the article

\begin{abstract}
In this work, we investigate the fuzzy Adomian decomposition method (ADM) and fuzzy variational iteration method (VIM) applied to solving fuzzy heat-like and wave-like equations with variable coefficients, in the sense of $\mathrm{gH}$-differentiability. The methods clearly are very efficient and powerful techniques in finding the solutions to the proposed equations. We also illustrate them by some examples. The results reveal that the methods are very effective, convenient, and accurate mathematical tools for solving fuzzy heat-like and wave-like equations with variable coefficients.
\end{abstract}

Keywords: Fuzzy numbers; Fuzzy-valued functions; gH-differentiability; Fuzzy Adomian decomposition method; Fuzzy variational iteration method; Fuzzy heat-like and wave-like equations

\section{Introduction}

The fuzzy set theory is a powerful tool for modeling uncertainty and processing vague or subjective information in mathematical models, which have been applied to a wide variety of real problems; for example, see $[4,17,19,20,56]$. Especially, the fuzzy differential equations (FDEs) are a very important topic from the theoretical point of view; some authors are interest in these works, for example, Fei [21] studied the existence and uniqueness of solutions for fuzzy random differential equations (FRDEs) with non-Lipschitz coefficients. The concept of differential equations in the fuzzy environment was introduced by Kaleva [42] in 1986. Kaleva [43] also presented the Cauchy problem for FDEs. Lopez [44] obtained comparison results for FDEs. Friedman and Kandel [45] introduced numerical solutions of FDEs. Mizukoshi et al. [46] studied the FDEs and the extension principle, as well as the applied point of view $[2,3,9]$; see, for example, $[14,15]$. The concept of the fuzzy derivative was initially proposed by Chang and Zadeh [16] in 1972. Dubois and Prade [18] introduced the extension principle. Other methods have been considered by Puri and Ralesu [51] and Goetshel and Voxman [23].

The fuzzy partial differential equations (FPDEs) attracted a great deal of attention among scientists and engineers because of their frequent involvement in the modeling of

(c) The Author(s) 2020. This article is licensed under a Creative Commons Attribution 4.0 International License, which permits use sharing, adaptation, distribution and reproduction in any medium or format, as long as you give appropriate credit to the original author(s) and the source, provide a link to the Creative Commons licence, and indicate if changes were made. The images or other third party material in this article are included in the article's Creative Commons licence, unless indicated otherwise in a credit line to the material. If material is not included in the article's Creative Commons licence and your intended use is not permitted by statutory regulation or exceeds the permitted use, you will need to obtain permission directly from the copyright holder. To view a copy of this licence, visit http://creativecommons.org/licenses/by/4.0/. 
numerous industrialized applications, for example, electromagnetic fields, heat and mass transfer, meteorology, static and dynamic of structures, biomechanics, and other areas of science and engineering. The FPDEs have considered by many researchers. Allahviranloo [7] used the Adomian decomposition method (ADM) for solving the fuzzy heat equation. Alikhani and Bahrami [6] presented the FPDEs under the cross product of fuzzy numbers. Jameel et al. [41] proposed a new approximation method for solving fuzzy heat equations. Stepnicka and Valasek [54] introduced the numerical solution of partial differential equations (PDEs) with the help of a fuzzy transform. Jameel [39] studied the semianalytical solution of the heat equation in a fuzzy environment. Allahviranloo et al. [10] have presented the fuzzy solutions for heat equation based on generalized Hukuhara differentiability. Osman et al. [47] used the differential transform method for solving fuzzy fractional wave equations under generalized Taylor's formula, gH-differentiability, and fuzzy Coputo's derivative.

The identification of the Lagrange multiplier is extremely very important in mathematical tools and can be optimized by using the variational theory. He[33] introduced a generalized variational principle and obtained a parameterized generalized variational principle for large deformation analysis of circular cylinders by the semiinverse method. He and Ain [36] established new promises and future challenges of fractal calculus. from two-scale thermodynamics to fractal variational principle. $\mathrm{He}$ [34] used the variational principle and periodic solution of the Kundu-Mukherjee-Naskar equation.

The Adomian decomposition method (ADM) has been lately intensively considered by scientists and engineers and studied for solving linear, nonlinear differential, and integral problems. Hamoud and Ghadle [28] successfully applied the ADM and the modified Laplace ADM to find approximate solutions of a nonlinear fractional Volterra-Fredholm integro-differential equation under Caputo's fractional derivative. Hamoud et al. [30] studied the behavior of approximate solutions of the Caputo fractional Volterra-Fredholm integro-differential equations. At the same time, several researchers used this method for solving many equations in the fuzzy environment; see $[1,13,27,48,49,53]$.

The variational iteration method (VIM) was originally proposed by He [32]. Later, several authors found VIM to be an effective way for solving some equations; see [5, 26, 29, 31, $35,37,38]$. Furthermore, in a fuzzy environment, several authors studied the VIM. Allahviranloo et al. [8] considered the fuzzy VIM for solving nonlinear fuzzy differential equations. Jameel [40] presented an approximate analytical solution for an initial value problem involving the fuzzy Duffing ordinary differential equation by using VIM. Hamoud et al. [25] studied the ADM, VIM, and homotopy analysis method for solving fuzzy VolterraFredholm integral equations. Zhang et al. [57] proposed a fuzzy model for dye removal and suggested to study a transport model of the direct textile industry wastewater. In this paper, we present a comparison of the fuzzy Adomian decomposition method and the fuzzy variational iteration method for solving fuzzy heat-like and wave-like equations with variable coefficients under the gH-differentiability.

This work is structured as follows. In Sect. 2, we recall some definitions of fuzzy numbers, fuzzy-valued functions, and strongly gH-differentiable functions. In Sect. 3, we propose the analysis of fuzzy heat-like and wave-like equations with variable coefficients under gH-differentiability by using the fuzzy ADM and VIM. In Sect. 4, we provide some examples to show the efficiency and simplicity of the ways they were developed and derived. Finally, a conclusion is given in Sect. 5. 


\section{Basic concepts}

The basic definition of fuzzy numbers is given in [58]. We denote the set of all real numbers by $R$ and the set of all fuzzy numbers on $R$ by $E^{1}$. A fuzzy number is a mapping $u: R \rightarrow[0,1]$ with the following properties:

1. $\tilde{u}$ is normal, that is, there exists $x_{0} \in R$ with $\tilde{u}\left(x_{0}\right)=1$;

2. $\tilde{u}$ is a convex fuzzy set (i.e., $\tilde{u}(\lambda x+(1-\lambda) y)) \geq \min \{\tilde{u}(x), \tilde{u}(y)\}, \forall \lambda \in[0,1], x, y \in R$;

3. $\tilde{u}$ is semicontinuous on $R$;

4. $\{x \in R: \tilde{u}(x)>0\}$ is compact, where $\bar{A}$ denotes the closure of $A$.

Definition 2.1 $([45,52,58])$ A fuzzy number $\tilde{u}$ in parametric from is a pair $(\underline{u}, \bar{u})$ of functions $\underline{u}(r), \bar{u}(r), 0 \leq r \leq 1$, satisfying the following requirements:

1. $\underline{u}_{r}$ is a bounded increasing left-continuous function,

2. $\bar{u}_{r}$ is a bounded decreasing left-continuous function,

3. $\underline{u}_{r} \leq \bar{u}(r), 0 \leq r \leq 1$.

Applying Zadeh's extension principle, the operation of addition on $E^{1}$ is defined by

$$
(\tilde{u}+\tilde{v})(x)=\sup _{y \in R} \min \{u(y), v(x-y)\}, \quad x \in R,
$$

and the scalar multiplication of fuzzy numbers is given by

$$
(k \odot \tilde{u})(x)= \begin{cases}\tilde{u}(x / k), & k>0, \\ \tilde{0}, & k=0,\end{cases}
$$

where $\tilde{0} \in E^{1}$.

The Hausdorff distance between fuzzy numbers is the map $d: E^{1} \times E^{1} \rightarrow[0,+\infty]$ given by

$$
d(\tilde{u}, \tilde{v})=\sup _{r \in[0,1]} \max \left\{\left|\underline{u}_{r}-\underline{v}_{r}\right|,\left|\bar{u}_{r}-\bar{v}_{r}\right|\right\}
$$

for $\tilde{u}=\left(\underline{u}_{r}, \bar{u}_{r}\right)$ and $\tilde{v}=\left(\underline{v}_{r}, \bar{v}_{r}\right) \subset R[11]$. It is easy to see that $d$ is a metric in $E^{1}$ and has the following properties [50]:

1. $\left(E^{1}, D\right)$ is a complete metric space,

2. $D(\tilde{u} \oplus \tilde{w}, \tilde{v} \oplus \tilde{w})=D(\tilde{u}, \tilde{v}), \forall \tilde{u}, \tilde{v}, \tilde{w} \in E^{1}$,

3. $D(\tilde{u} \oplus \tilde{v}, \tilde{w} \oplus \tilde{e}) \leq D(\tilde{u}, \tilde{w})+D(\tilde{v}, \tilde{e}), \forall \tilde{u}, \tilde{v}, \tilde{w}, \tilde{e} \in E^{1}$,

4. $D(k \odot \tilde{u}, k \odot \tilde{v})=|k| D(\tilde{u}, \tilde{v}), \forall \tilde{u}, \tilde{v} \in E^{1}, k \in R$.

Note that functions $\tilde{f}: A \rightarrow E^{1}, A \subseteq R$, are further called fuzzy-valued functions, whereas functions $\tilde{f}: A \rightarrow R, A \subseteq R$, are called real-valued functions (or crisp functions). The $r$-level representation of fuzzy-valued function $\tilde{f}$ can be expressed by $\tilde{f}(x ; r)=$ $[f(x ; r), \bar{f}(x ; r)]$ for $x \in A \subseteq R$ and $0 \leq r \leq 1$.

Definition 2.2 ([42]) A fuzzy-valued function $\tilde{f}:[a, b] \rightarrow E^{1}$ is said to satisfy condition (gH) on $[a, b]$ if for any $x_{1}<x_{2} \in[a, b]$, there exists $\tilde{u} \in E^{1}$ such that $\tilde{f}\left(x_{2}\right)=\tilde{f}\left(x_{1}\right)+\tilde{u}$. We call $\tilde{u}$ the gH-difference of $\tilde{f}\left(x_{2}\right)$ and $\tilde{f}\left(x_{1}\right)$, denoted $\tilde{f}\left(x_{2}\right) \ominus_{g H} \tilde{f}\left(x_{1}\right)$. For brevity, we always assume that it satisfies condition $(\mathrm{gH})$ when we deal with the subtraction of fuzzy numbers. 
Definition $2.3([12,55])$ The generalized Hukuhara difference of two fuzzy numbers $\tilde{u}, \tilde{v} \in E^{1}$ is defined as follows:

$$
\tilde{u} \ominus_{g H} \tilde{v}=\tilde{w} \Leftrightarrow \begin{cases}\text { (i) } & \tilde{u}=\tilde{v} \oplus \tilde{w}, \quad \text { or } \\ \text { (ii) } & \tilde{v}=\tilde{u} \oplus(-\tilde{w}) .\end{cases}
$$

In terms of the $r$-levels, we get $\left[\tilde{u} \ominus_{g H} \tilde{v}\right]=\left[\min \left\{\underline{u}_{r}-\underline{v}_{r}, \bar{u}_{r}-\bar{v}_{r}\right\}, \max \left\{\underline{u}_{r}-\underline{v}_{r}, \bar{u}_{r}-\bar{v}_{r}\right\}\right]$, and if the H-difference exists, then $\tilde{u} \ominus \tilde{v}=\tilde{u} \ominus_{g H} \tilde{v}$; conditions for the existence of $\tilde{w}=$ $\tilde{u} \ominus_{g H} \tilde{v} \in E^{1}$ are

Case (i) $\left\{\begin{array}{l}\underline{w}_{r}=\underline{u}_{r}-\underline{v}_{r} \quad \text { and } \quad \bar{w}_{r}=\bar{u}_{r}-\bar{v}_{r}, \quad \forall r \in[0,1], \\ \text { with } \underline{w}_{r} \text { increasing, } \bar{w}_{r} \text { decreasing, } \underline{w}_{r} \leq \bar{w}_{r},\end{array}\right.$

Case (ii) $\left\{\begin{array}{l}\underline{w}_{r}=\bar{u}_{r}-\bar{v}_{r} \quad \text { and } \quad \bar{w}_{r}=\underline{u}_{r}-\underline{v}_{r}, \quad \forall r \in[0,1], \\ \text { with } \underline{w}_{r} \text { increasing, } \bar{w}_{r} \text { decreasing, } \underline{w}_{r} \leq \bar{w}_{r} .\end{array}\right.$

It is easy to show that (i) and (ii) are both valid if and only if $\tilde{w}$ is a crisp number. In this case, it is possible that the gH-difference of two fuzzy numbers does not exist. To address this shortcoming, a new difference of fuzzy numbers was introduced in [12].

Definition 2.4 ([11]) Let $\tilde{u}(x, t): D \rightarrow E^{1}$ and $\left(x_{0}, t\right) \in D$. We say that $\tilde{u}$ is strongly generalized Hukuhara differentiable on $\left(x_{0}, t\right)$ (gH-differentiable for short) if there exists an element $\left.\frac{\partial \tilde{u}}{\partial x}\right|_{\left(x_{0}, t\right)} \in E^{1}$ such that

(i) for all $h>0$ sufficiently small, $\tilde{u}\left(x_{0}+h, t\right) \ominus_{g H} \tilde{u}\left(x_{0}, t\right), \tilde{u}\left(x_{0}, t\right) \ominus_{g H} \tilde{u}\left(x_{0}-h, t\right)$ and the limits (in the metric $D$ )

$$
\lim _{h \rightarrow 0+} \frac{\tilde{u}\left(x_{0}+h, t\right) \ominus_{g H} \tilde{u}\left(x_{0}, t\right)}{h}=\lim _{h \rightarrow 0+}=\frac{\tilde{u}\left(x_{0}, t\right) \ominus_{g H} \tilde{u}\left(x_{0}-h, t\right)}{h}=\left.\frac{\partial \tilde{u}}{\partial x_{g H}}\right|_{\left(x_{0}, t\right)},
$$

or

(ii) for all $h>0$ sufficiently small, there exist $\tilde{u}\left(x_{0}, t\right) \ominus_{g H} \tilde{u}\left(x_{0}+h, t\right)$,

$\tilde{u}\left(x_{0}-h, t\right) \ominus_{g H} \tilde{u}\left(x_{0}, t\right)$ and the limits

$$
\lim _{h \rightarrow 0+} \frac{\tilde{u}\left(x_{0}, t\right) \ominus_{g H} \tilde{u}\left(x_{0}+h, t\right)}{-h}=\lim _{h \rightarrow 0+} \frac{\tilde{u}\left(x_{0}-h, t\right) \ominus_{g H} \tilde{u}\left(x_{0}, t\right)}{-h}=\left.\frac{\partial \tilde{u}}{\partial x_{g H}}\right|_{\left(x_{0}, t\right)},
$$

or

(iii) for all $h>0$ sufficiently small, there exist $\tilde{u}\left(x_{0}+h, t\right) \ominus_{g H} \tilde{u}\left(x_{0}, t\right)$,

$\tilde{u}\left(x_{0}-h, t\right) \ominus_{g H} \tilde{u}\left(x_{0}, t\right)$ and the limits

$$
\lim _{h \rightarrow 0+} \frac{\tilde{u}\left(x_{0}+h, t\right) \ominus_{g H} \tilde{u}\left(x_{0}, t\right)}{h}=\lim _{h \rightarrow 0+} \frac{\tilde{u}\left(x_{0}-h, t\right) \ominus_{g H} \tilde{u}\left(x_{0}, t\right)}{-h}=\left.\frac{\partial \tilde{u}}{\partial x_{g H}}\right|_{\left(x_{0}, t\right)},
$$

or

(iv) for all $h>0$ sufficiently small, there exist $\tilde{u}\left(x_{0}, t\right) \ominus_{g H} \tilde{u}\left(x_{0}+h, t\right)$,

$\tilde{u}\left(x_{0}, t\right) \ominus_{g H} \tilde{u}\left(x_{0}-h, t\right)$ and the limits

$$
\lim _{h \rightarrow 0+} \frac{\tilde{u}\left(x_{0}, t\right) \ominus_{g H} \tilde{u}\left(x_{0}+h, t\right)}{-h}=\lim _{h \rightarrow 0+} \frac{\tilde{u}\left(x_{0}, t\right) \ominus_{g H} \tilde{u}\left(x_{0}-h, t\right)}{h}=\left.\frac{\partial \tilde{u}}{\partial x_{g H}}\right|_{\left(x_{0}, t\right)} .
$$


Definition 2.5 ([24]) Let $\tilde{u}(x, t): D \rightarrow E^{1}$ and $\left(x_{0}, t\right) \in D$. We define the $n$ th-order derivative of $\tilde{u}$ as follows: we say that $\tilde{u}$ is strongly generalized Hukuhara differentiable of the $n$th order at $\left(x_{0}, t\right)$ (gH-differentiable for short) if there exists elements $\left.\frac{\partial^{5} \tilde{u}}{\partial x^{s}}\right|_{\left(x_{0}, t\right)} \in E^{1}$, $s=1,2, \ldots, n$, such that

(i) for all $h>0$ sufficiently small, there exist $\tilde{u}^{(s-1)}\left(x_{0}+h, t\right) \ominus_{g H} \tilde{u}^{(s-1)}\left(x_{0}, t\right)$, $\tilde{u}^{(s-1)}\left(x_{0}, t\right) \ominus_{g H} \tilde{u}^{(s-1)}\left(x_{0}-h, t\right)$ and the limits (in the metric $D$ )

$$
\begin{aligned}
& \lim _{h \rightarrow 0+} \frac{\tilde{u}^{(s-1)}\left(x_{0}+h, t\right) \ominus_{g H} \tilde{u}^{(s-1)}\left(x_{0}, t\right)}{h} \\
& \quad=\lim _{h \rightarrow 0+}=\frac{\tilde{u}^{(s-1)}\left(x_{0}, t\right) \ominus_{g H} \tilde{u}^{(s-1)}\left(x_{0}-h, t\right)}{h}=\left.\left.\frac{\partial^{s} \tilde{u}}{\partial x^{s}}\right|_{g H}\right|_{\left(x_{0}, t\right)},
\end{aligned}
$$

or

(ii) for all $h>0$ sufficiently small, there exist $\tilde{u}^{(s-1)}\left(x_{0}, t\right) \ominus_{g H} \tilde{u}^{(s-1)}\left(x_{0}+h, t\right)$, $\tilde{u}^{(s-1)}\left(x_{0}-h, t\right) \ominus_{g H} \tilde{u}^{(s-1)}\left(x_{0}, t\right)$ and the limits

$$
\begin{aligned}
& \lim _{h \rightarrow 0+} \frac{\tilde{u}^{(s-1)}\left(x_{0}, t\right) \ominus_{g H} \tilde{u}^{(s-1)}\left(x_{0}+h, t\right)}{-h} \\
& \quad=\lim _{h \rightarrow 0+} \frac{\tilde{u}^{(s-1)}\left(x_{0}-h, t\right) \ominus_{g H} \tilde{u}^{(s-1)}\left(x_{0}, t\right)}{-h}=\left.\left.\frac{\partial^{s} \tilde{u}}{\partial x^{s}}\right|_{g H}\right|_{\left(x_{0}, t\right)},
\end{aligned}
$$

or

(iii) for all $h>0$ sufficiently small, there exist $\tilde{u}^{(s-1)}\left(x_{0}+h, t\right) \ominus_{g H} \tilde{u}^{(s-1)}\left(x_{0}, t\right)$, $\tilde{u}^{(s-1)}\left(x_{0}-h, t\right) \ominus_{g H} \tilde{u}^{(s-1)}\left(x_{0}, t\right)$ and the limits

$$
\begin{aligned}
& \lim _{h \rightarrow 0+} \frac{\tilde{u}^{(s-1)}\left(x_{0}+h, t\right) \ominus_{g H} \tilde{u}^{(s-1)}\left(x_{0}, t\right)}{h} \\
& \quad=\lim _{h \rightarrow 0+} \frac{\tilde{u}^{(s-1)}\left(x_{0}-h, t\right) \ominus_{g H} \tilde{u}^{(s-1)}\left(x_{0}, t\right)}{-h}=\left.\frac{\partial^{s} \tilde{u}}{\partial x^{s}}{ }_{g H}\right|_{\left(x_{0}, t\right)},
\end{aligned}
$$

or

(iv) for all $h>0$ sufficiently small, there exist $\tilde{u}^{(s-1)}\left(x_{0}, t\right) \ominus_{g H} \tilde{u}^{(s-1)}\left(x_{0}+h, t\right)$, $\tilde{u}^{(s-1)}\left(x_{0}, t\right) \ominus_{g H} \tilde{u}^{(s-1)}\left(x_{0}-h, t\right)$ and the limits

$$
\begin{aligned}
& \lim _{h \rightarrow 0+} \frac{\tilde{u}^{(s-1)}\left(x_{0}, t\right) \ominus_{g H} \tilde{u}^{(s-1)}\left(x_{0}+h, t\right)}{-h} \\
& \quad=\lim _{h \rightarrow 0+} \frac{\tilde{u}^{(s-1)}\left(x_{0}, t\right) \ominus_{g H} \tilde{u}^{(s-1)}\left(x_{0}-h, t\right)}{h}=\left.\frac{\partial^{s} \tilde{u}}{\partial x^{s}}\right|_{\left(x_{0}, t\right)} .
\end{aligned}
$$

\section{Fuzzy heat-like and wave-like equations with variable coefficients}

In this section, we analyze fuzzy heat-like and wave-like equations with variable coefficients under gH-differentiability by using the fuzzy Adomian decomposition method and fuzzy variational iteration method as follows.

First, we consider the fuzzy heat-like equation of the form

$$
\begin{aligned}
\tilde{u}_{t}=f(x, y, z) \odot \tilde{u}_{\mathrm{xx}} \oplus g(x, y, z) \odot \tilde{u}_{\mathrm{yy}} \oplus h(x, y, z) \odot \tilde{u}_{\mathrm{zz}}, \\
\quad 0<x<a, 0<y<b, 0<z<c, t>0, f(x, y, z) \geq 0, g(x, y, z) \geq 0, h(x, y, z) \geq 0,
\end{aligned}
$$


with the Neumann boundary conditions

$$
\begin{aligned}
& \tilde{u}_{\mathrm{x}}(0, y, z, t)=\tilde{f}_{1}(y, z, t), \quad \tilde{u}_{\mathrm{x}}(a, y, z, t)=\tilde{f}_{2}(y, z, t), \\
& \tilde{u}_{\mathrm{y}}(x, 0, z, t)=\tilde{g}_{1}(x, z, t), \quad \tilde{u}_{\mathrm{y}}(x, b, z, t)=\tilde{g}_{2}(x, z, t), \\
& \tilde{u}_{\mathrm{z}}(x, y, 0, t)=\tilde{h}_{1}(x, y, t), \quad \tilde{u}_{\mathrm{z}}(x, y, c, t)=\tilde{h}_{2}(x, y, t),
\end{aligned}
$$

and the initial conditions

$$
\tilde{u}(x, y, z, 0)=\tilde{\varphi}(x, y, z) .
$$

Second, we consider the fuzzy wave-like equation of the form

$$
\begin{aligned}
\tilde{u}_{t t} & =f(x, y, z) \odot \tilde{u}_{\mathrm{xx}} \oplus g(x, y, z) \odot \tilde{u}_{\mathrm{yy}} \oplus h(x, y, z) \odot \tilde{u}_{\mathrm{zz}}, \\
& 0<x<a, 0<y<b, 0<z<c, t>0, f(x, y, z) \geq 0, g(x, y, z) \geq 0, h(x, y, z) \geq 0,
\end{aligned}
$$

with the Neumann boundary conditions

$$
\begin{array}{ll}
\tilde{u}_{\mathrm{x}}(0, y, z, t)=\tilde{f}_{1}(y, z, t), \quad \tilde{u}_{\mathrm{x}}(a, y, z, t)=\tilde{f}_{2}(y, z, t), \\
\tilde{u}_{\mathrm{y}}(x, 0, z, t)=\tilde{g}_{1}(x, z, t), \quad \tilde{u}_{\mathrm{y}}(x, b, z, t)=\tilde{g}_{2}(x, z, t), \\
\tilde{u}_{\mathrm{z}}(x, y, 0, t)=\tilde{h}_{1}(x, y, t), \quad & \tilde{u}_{\mathrm{z}}(x, y, c, t)=\tilde{h}_{2}(x, y, t),
\end{array}
$$

and the initial conditions

$$
\tilde{u}(x, y, z, 0)=\tilde{\varphi}(x, y, z), \quad \tilde{u}_{t}(x, y, z, 0)=\tilde{\vartheta}(x, y, z) .
$$

\subsection{The fuzzy Adomian decomposition method}

We derive the main algorithms of the fuzzy Adomian decomposition method for fuzzy heat-like and wave-like equations as follows.

\subsubsection{Fuzzy heat-like equations}

We consider the following parametric of fuzzy heat-like equation (3.1) of the form:

$$
\begin{aligned}
\underline{u}_{t}=f(x, y, z) \underline{u}_{\mathrm{xx}}+g(x, y, z) \underline{u}_{\mathrm{yy}}+h(x, y, z) \underline{u}_{\mathrm{zz}}, \\
\quad 0<x<a, 0<y<b, 0<z<c, t>0, f(x, y, z) \geq 0, g(x, y, z) \geq 0, h(x, y, z) \geq 0, \\
\bar{u}_{t}=f(x, y, z) \bar{u}_{\mathrm{xx}}+g(x, y, z) \bar{u}_{\mathrm{yy}}+h(x, y, z) \bar{u}_{\mathrm{zz}}, \\
\quad 0<x<a, 0<y<b, 0<z<c, t>0, f(x, y, z) \geq 0, g(x, y, z) \geq 0, h(x, y, z) \geq 0 .
\end{aligned}
$$

In an operator form, equations (3.8) and (3.9) can be written as

$$
\begin{aligned}
& L_{t} \underline{u}(r)=\left[f(x, y, z) \underline{u}_{\mathrm{xx}}+g(x, y, z) \underline{u}_{\mathrm{yy}}+h(x, y, z) \underline{u}_{\mathrm{zz}}\right](r), \\
& 0<x<a, 0<y<b, 0<z<c, 0<\alpha<1, t>0, \\
& L_{t} \bar{u}(r)= {\left[f(x, y, z) \bar{u}_{\mathrm{xx}}+g(x, y, z) \bar{u}_{\mathrm{yy}}+h(x, y, z) \bar{u}_{\mathrm{zz}}\right](r), } \\
& 0<x<a, 0<y<b, 0<z<c, 0<\alpha<1, t>0,
\end{aligned}
$$


where the differential operator $L_{t}$ is

$$
L_{t}=\frac{\partial}{\partial t}
$$

Assuming that $L_{t}$ is invertible, the inverse operator $L_{t}^{-1}$ is given by the integral

$$
L_{t}^{-1}(\cdot)=\int_{0}^{t}(\cdot) d s .
$$

Applying the inverse operator $L_{t}^{-1}$ to equations (3.10) and (3.11) and using the initial condition (3.4), we obtain

$$
\begin{aligned}
& \underline{u}(x, y, z, t ; r)=\underline{\varphi}(x, y, z ; r)+L_{t}^{-1}\left[f(x, y, z) \underline{u}_{x x}+g(x, y, z) \underline{u}_{y y}+h(x, y, z) \underline{u}_{z z}\right](r), \\
& \bar{u}(x, y, z, t ; r)=\bar{\varphi}(x, y, z ; r)+L_{t}^{-1}\left[f(x, y, z) \bar{u}_{x x}+g(x, y, z) \bar{u}_{y y}+h(x, y, z) \bar{u}_{z z}\right](r) .
\end{aligned}
$$

The fuzzy ADM consists of representing the solution for

$$
\tilde{u}(x, y, z, t)(r)=[\underline{u}(x, y, z, t)(r), \bar{u}(x, y, z, t)(r)]
$$

by an infinite sum of components as follows:

$$
\begin{aligned}
& \underline{u}(x, y, z, t ; r)=\sum_{m=0}^{\infty} \underline{u}_{m}(x, y, z, t ; r), \\
& \bar{u}(x, y, z, t ; r)=\sum_{m=0}^{\infty} \bar{u}_{m}(x, y, z, t ; r),
\end{aligned}
$$

where the fuzzy components $\tilde{u}_{m}(x, y, z, t ; r)=\left[\underline{u}_{m}(x, y, z, t ; r), \bar{u}_{m}(x, y, z, t ; r)\right]$ will be recursively determined. Applying (3.16) to both sides of (3.13) gives

$$
\begin{aligned}
\sum_{m=0}^{\infty} \underline{u}_{m}(x, y, z, t ; r)= & \underline{\varphi}(x, y, z ; r)+L_{t}^{-1}\left[f\left(\sum_{m=0}^{\infty}\left(\underline{u}_{m}(x, y, z, t ; r)\right)\right)_{\mathrm{xx}}\right. \\
& \left.+g\left(\sum_{m=0}^{\infty}\left(\underline{u}_{m}(x, y, z, t ; r)\right)\right)_{\mathrm{yy}}+h\left(\sum_{m=0}^{\infty}\left(\underline{u}_{m}(x, y, z, t ; r)\right)\right)_{\mathrm{zz}}\right]
\end{aligned}
$$

and

$$
\begin{aligned}
\sum_{m=0}^{\infty} \bar{u}_{m}(x, y, z, t ; r)= & \bar{\varphi}(x, y, z ; r)+L_{t}^{-1}\left[f\left(\sum_{m=0}^{\infty}\left(\bar{u}_{m}(x, y, z, t ; r)\right)\right)_{\mathrm{xx}}\right. \\
& \left.+g\left(\sum_{m=0}^{\infty}\left(\bar{u}_{m}(x, y, z, t ; r)\right)\right)_{\mathrm{yy}}+h\left(\sum_{m=0}^{\infty}\left(\bar{u}_{m}(x, y, z, t ; r)\right)\right)_{\mathrm{zz}}\right] .
\end{aligned}
$$

Next, the fuzzy ADM identifies the zeroth component $\tilde{u}_{0}(x, y, z, t ; r)=\left[\underline{u}_{0}(x, y, z, t ; r)\right.$, $\left.\bar{u}_{0}(x, y, z, t ; r)\right]$ by all terms that arise from the initial condition and from integrating the source term if exists. To determine the components $\tilde{u}_{m}(x, y, z, t ; r)=\left[\underline{u}_{m}(x, y, z, t ; r), \bar{u}_{m}(x, y\right.$, 
$z, t ; r)], m \geq 1$, of the solution $\tilde{u}(x, y, z, t ; r)$, fuzzy ADM formally introduces the recursive relation as follows:

$$
\begin{aligned}
& \underline{u}_{0}(x, y, z, t ; r)=\underline{\varphi}(x, y, z ; r), \\
& \underline{u}_{m+1}(x, y, z, t ; r) \\
& \quad=L_{t}^{-1}\left[f\left(\underline{u}_{m}(x, y, z, t ; r)\right)_{\mathrm{xx}}+g\left(\underline{u}_{m}(x, y, z, t ; r)\right)_{\mathrm{yy}}+h\left(\underline{u}_{m}(x, y, z, t ; r)\right)_{\mathrm{zz}}\right] \\
& \quad m \geq 0, \\
& \bar{u}_{0}(x, y, z, t ; r)=\bar{\varphi}(x, y, z ; r), \\
& \bar{u}_{m+1}(x, y, z, t ; r) \\
& \quad=L_{t}^{-1}\left[f\left(\bar{u}_{m}(x, y, z, t ; r)\right)_{\mathrm{xx}}+g\left(\bar{u}_{m}(x, y, z, t ; r)\right)_{\mathrm{yy}}+h\left(\bar{u}_{m}(x, y, z, t ; r)\right)_{\mathrm{zz}}\right], \\
& \quad m \geq 0 .
\end{aligned}
$$

Thus we can recurrently determine every component of the series $\sum_{m=0}^{\infty} \tilde{u}_{m}(x, y, z, t ; r)$ and so obtain the series solution of $\tilde{u}(x, y, z, t ; r)$.

\subsubsection{Inhomogeneous fuzzy heat-like equations}

We consider the inhomogeneous fuzzy heat-like equation of the form

$$
\begin{aligned}
\tilde{u}_{t}=\tilde{\tau}(x, y, z) \oplus f(x, y, z) \odot \tilde{u}_{\mathrm{xx}} \oplus g(x, y, z) \odot \tilde{u}_{\mathrm{yy}} \oplus h(x, y, z) \odot \tilde{u}_{\mathrm{zz}}, \\
\quad 0<x<a, 0<y<b, 0<z<c, t>0, f(x, y, z) \geq 0, g(x, y, z) \geq 0, h(x, y, z) \geq 0,
\end{aligned}
$$

The parametric form of (3.21) is

$$
\begin{aligned}
L_{t} \underline{u}(r) & =\underline{\tau}(x, y, z ; r)+\left[f(x, y, z) \underline{u}_{\mathrm{xx}}+g(x, y, z) \underline{u}_{\mathrm{yy}}+h(x, y, z) \underline{u}_{\mathrm{zz}}\right](r), \\
0<x & <a, 0<y<b, 0<z<c, 0<\alpha<1, t>0, \\
L_{t} \bar{u}(r) & =\bar{\tau}(x, y, z ; r)+\left[f(x, y, z) \bar{u}_{\mathrm{xx}}+g(x, y, z) \bar{u}_{\mathrm{yy}}+h(x, y, z) \bar{u}_{\mathrm{zz}}\right](r), \\
0<x & <a, 0<y<b, 0<z<c, 0<\alpha<1, t>0,
\end{aligned}
$$

where

$$
L_{t}=\frac{\partial}{\partial t} .
$$

Assuming that $L_{t}$ is invertible, the inverse operator $L_{t}^{-1}$ is given by the integral

$$
L_{t}^{-1}(\cdot)=\int_{0}^{t}(\cdot) d s
$$

Applying the inverse operator $L_{t}^{-1}$ to (3.22) and (3.23), by the initial condition (3.4) we obtain

$$
\underline{u}(x, y, z, t ; r)=\underline{\tau}(x, y, z ; r) t+L_{t}^{-1}\left[f(x, y, z) \underline{u}_{x x}+g(x, y, z) \underline{u}_{y y}+h(x, y, z) \underline{u}_{z z}\right](r),
$$




$$
\bar{u}(x, y, z, t ; r)=\bar{\tau}(x, y, z ; r) t+L_{t}^{-1}\left[f(x, y, z) \bar{u}_{x x}+g(x, y, z) \bar{u}_{y y}+h(x, y, z) \bar{u}_{z z}\right](r) .
$$

The fuzzy ADM assumes a series solution for

$$
\tilde{u}(x, y, z, t ; r)=[\underline{u}(x, y, z, t ; r), \bar{u}(x, y, z, t ; r)]
$$

given by an infinite sum of components as follows:

$$
\begin{aligned}
& \underline{u}(x, y, z, t ; r)=\sum_{m=0}^{\infty} \underline{u}_{m}(x, y, z, t ; r), \\
& \bar{u}(x, y, z, t ; r)=\sum_{m=0}^{\infty} \bar{u}_{m}(x, y, z, t ; r),
\end{aligned}
$$

where the fuzzy components $\tilde{u}_{m}(x, y, z, t ; r)=\left[\underline{u}_{m}(x, y, z, t ; r), \bar{u}_{m}(x, y, z, t ; r)\right]$ will be recursively determined. Substituting (3.28) into both sides of (3.25) gives

$$
\begin{aligned}
\sum_{m=0}^{\infty} \underline{u}_{m}(x, y, z, t ; r)= & \underline{\tau}(x, y, z ; r) t+L_{t}^{-1}\left[f\left(\sum_{m=0}^{\infty}\left(\underline{u}_{m}(x, y, z, t ; r)\right)\right)_{\mathrm{xx}}\right. \\
& \left.+g\left(\sum_{m=0}^{\infty}\left(\underline{u}_{m}(x, y, z, t ; r)\right)\right)_{\mathrm{yy}}+h\left(\sum_{m=0}^{\infty}\left(\underline{u}_{m}(x, y, z, t ; r)\right)\right)_{\mathrm{zz}}\right]
\end{aligned}
$$

and

$$
\begin{aligned}
\sum_{m=0}^{\infty} \bar{u}_{m}(x, y, z, t ; r)= & \bar{\tau}(x, y, z ; r) t+L_{t}^{-1}\left[f\left(\sum_{m=0}^{\infty}\left(\bar{u}_{m}(x, y, z, t ; r)\right)\right)_{\mathrm{xx}}\right. \\
& \left.+g\left(\sum_{m=0}^{\infty}\left(\bar{u}_{m}(x, y, z, t ; r)\right)\right)_{\mathrm{yy}}+h\left(\sum_{m=0}^{\infty}\left(\bar{u}_{m}(x, y, z, t ; r)\right)\right)_{\mathrm{zz}}\right]
\end{aligned}
$$

Following the fuzzy ADM, we identify the zeroth component $\tilde{u}_{0}(x, y, z, t ; r)=\left[\underline{u}_{0}(x, y, z, t\right.$; $\left.r), \bar{u}_{0}(x, y, z, t ; r)\right]$ by all terms that arise from the initial condition and from integrating the source term if exists. To determine the components $\tilde{u}_{m}(x, y, z, t ; r), m \geq 1$, of the solution $\tilde{u}(x, y, z, t ; r)$, the fuzzy ADM formally introduces the recursive relation

$$
\begin{aligned}
& \underline{u}_{0}(x, y, z, t ; r)=\underline{\tau}(x, y, z ; r) t, \\
& \underline{u}_{m+1}(x, y, z, t)(r) \\
& \quad=L_{t}^{-1}\left[f\left(\underline{u}_{m}(x, y, z, t ; r)\right)_{\mathrm{xx}}+g\left(\underline{u}_{m}(x, y, z, t ; r)\right)_{\mathrm{yy}}+h\left(\underline{u}_{m}(x, y, z, t ; r)\right)_{\mathrm{zz}}\right], \\
& \quad m \geq 0, \\
& \quad \bar{u}_{0}(x, y, z, t ; r)=\bar{\tau}(x, y, z ; r) t, \\
& \bar{u}_{m+1}(x, y, z, t)(r) \\
& \quad=L_{t}^{-1}\left[f\left(\bar{u}_{m}(x, y, z, t ; r)\right)_{\mathrm{xx}}+g\left(\bar{u}_{m}(x, y, z, t ; r)\right)_{\mathrm{yy}}+h\left(\bar{u}_{m}(x, y, z, t ; r)\right)_{\mathrm{zz}}\right], \\
& \quad m \geq 0 .
\end{aligned}
$$




\subsubsection{Fuzzy wave-like equations}

We consider the fuzzy wave-like equation (3.5) of the form

$$
\begin{gathered}
\underline{u}_{t t}=\left[f(x, y, z) \underline{u}_{\mathrm{xx}}+g(x, y, z) \underline{u}_{\mathrm{yy}}+h(x, y, z) \underline{u}_{\mathrm{zz}}\right](r), \\
0<x<a, 0<y<b, 0<z<c, 1<\alpha \leq 2, t>0, \\
\bar{u}_{t t}=\left[f(x, y, z) \bar{u}_{\mathrm{xx}}+g(x, y, z) \bar{u}_{\mathrm{yy}}+h(x, y, z) \bar{u}_{\mathrm{zz}}\right](r), \\
0<x<a, 0<y<b, 0<z<c, 1<\alpha \leq 2, t>0 .
\end{gathered}
$$

In an operator form, equations (3.33) and (3.34) become

$$
\begin{aligned}
& L_{t} \underline{u}(r)=\left[f(x, y, z) \underline{u}_{\mathrm{xx}}+g(x, y, z) \underline{u}_{\mathrm{yy}}+h(x, y, z) \underline{u}_{\mathrm{zz}}\right](r), \\
& L_{t} \bar{u}(r)=\left[f(x, y, z) \bar{u}_{\mathrm{xx}}+g(x, y, z) \bar{u}_{\mathrm{yy}}+h(x, y, z) \bar{u}_{\mathrm{zz}}\right](r),
\end{aligned}
$$

where

$$
L_{t}=\frac{\partial^{2}}{\partial t^{2}}
$$

Assuming that $L_{t}$ is invertible, the inverse operator $L_{t}^{-1}$ is given by the integral

$$
L_{t}^{-1}(\cdot)=\int_{0}^{t} \int_{0}^{t}(\cdot) d s d s
$$

Applying the inverse operator $L_{t}^{-1}$ to both sides of equations (3.35) and (3.36), by the initial conditions of equation (3.7) we have

$$
\begin{aligned}
\underline{u}(x, y, z, t ; r)= & \underline{\varphi}(x, y, z ; r)+t \underline{\vartheta}(x, y, z ; r) \\
& +L_{t}^{-1}\left[f(x, y, z) \underline{u}_{x x}+g(x, y, z) \underline{u}_{y y}+h(x, y, z) \underline{u}_{z z}\right](r), \\
\bar{u}(x, y, z, t ; r)= & \bar{\varphi}(x, y, z ; r)+t \bar{\vartheta}(x, y, z ; r) \\
& +L_{t}^{-1}\left[f(x, y, z) \bar{u}_{x x}+g(x, y, z) \bar{u}_{y y}+h(x, y, z) \bar{u}_{z z}\right](r) .
\end{aligned}
$$

The fuzzy ADM assumes a series solution for

$$
\tilde{u}(x, y, z, t ; r)=[\underline{u}(x, y, z, t ; r), \bar{u}(x, y, z, t ; r)],
$$

given by an infinite sum of components as follows:

$$
\begin{aligned}
& \underline{u}(x, y, z, t ; r)=\sum_{m=0}^{\infty} \underline{u}_{m}(x, y, z, t ; r), \\
& \bar{u}(x, y, z, t ; r)=\sum_{m=0}^{\infty} \bar{u}_{m}(x, y, z, t ; r),
\end{aligned}
$$


where the fuzzy components $\tilde{u}_{m}(x, y, z, t ; r)=\left[\underline{u}_{m}(x, y, z, t ; r), \bar{u}_{m}(x, y, z, t ; r)\right]$ will be repeatedly identified. Applying (3.41) to both sides of (3.38) gives

$$
\begin{aligned}
\sum_{m=0}^{\infty} \underline{u}_{m}(x, y, z, t ; r)= & \underline{\varphi}(x, y, z ; r)+t \underline{\vartheta}(x, y, z ; r)+L_{t}^{-1}\left[f\left(\sum_{m=0}^{\infty}\left(\underline{u}_{m}(x, y, z, t ; r)\right)\right)_{\mathrm{xx}}\right. \\
& \left.+g\left(\sum_{m=0}^{\infty}\left(\underline{u}_{m}(x, y, z, t ; r)\right)\right)_{\mathrm{yy}}+h\left(\sum_{m=0}^{\infty}\left(\underline{u}_{m}(x, y, z, t ; r)\right)\right)_{\mathrm{zz}}\right]
\end{aligned}
$$

and

$$
\begin{aligned}
\sum_{m=0}^{\infty} \bar{u}_{m}(x, y, z, t ; r)= & \bar{\varphi}(x, y, z ; r)+t \bar{\vartheta}(x, y, z, t ; r)+L_{t}^{-1}\left[f\left(\sum_{m=0}^{\infty}\left(\bar{u}_{m}(x, y, z, t ; r)\right)\right)_{\mathrm{xx}}\right. \\
& \left.+g\left(\sum_{m=0}^{\infty}\left(\bar{u}_{m}(x, y, z, t ; r)\right)\right)_{\mathrm{yy}}+h\left(\sum_{m=0}^{\infty}\left(\bar{u}_{m}(x, y, z, t ; r)\right)\right)_{\mathrm{zz}}\right] .
\end{aligned}
$$

To specify the components $\tilde{u}_{m}(x, y, z, t ; r), m \geq 0$, of the solution $\tilde{u}(x, y, z, t ; r)$, the fuzzy $\mathrm{ADM}$ officially introduces the recursive relationship

$$
\begin{aligned}
& \underline{u}_{0}(x, y, z, t ; r)=\underline{\varphi}(x, y, z ; r)+t \underline{\vartheta}(x, y, z ; r), \\
& \underline{u}_{m+1}(x, y, z, t ; r) \\
& \quad=L_{t}^{-1}\left[f\left(\underline{u}_{m}(x, y, z, t ; r)\right)_{\mathrm{xx}}+g\left(\underline{u}_{m}(x, y, z, t ; r)\right)_{\mathrm{yy}}+h\left(\underline{u}_{m}(x, y, z, t ; r)\right)_{\mathrm{zz}}\right], \\
& \quad m \geq 0 \\
& \bar{u}_{0}(x, y, z, t ; r)=\bar{\varphi}(x, y, z ; r)+t \bar{\vartheta}(x, y, z ; r), \\
& \bar{u}_{m+1}(x, y, z, t ; r) \\
& \quad=L_{t}^{-1}\left[f\left(\bar{u}_{m}(x, y, z, t ; r)\right)_{\mathrm{xx}}+g\left(\bar{u}_{m}(x, y, z, t ; r)\right)_{\mathrm{yy}}+h\left(\bar{u}_{m}(x, y, z, t ; r)\right)_{\mathrm{zz}}\right], \\
& \quad m \geq 0 .
\end{aligned}
$$

With this relationship, we easily obtain the components $\tilde{u}_{m}(x, y, z, t ; r), m \geq 0$. This, in turn, leads to a solution in the form of a series, but the solution is in a closed-form, which directly follows if there is an exact solution.

\subsection{The fuzzy variational iteration method}

We derive the main algorithms of the fuzzy variational iteration method considering the following general fuzzy partial differential equation:

$$
L_{t} \tilde{u} \oplus L_{x} \tilde{u} \oplus L_{y} \tilde{u} \oplus L_{z} \tilde{u} \oplus N \tilde{u}=\tilde{g}(x, y, z, t)
$$

where $L_{t}, L_{x}, L_{y}$, and $L_{z}$ are linear operators of $t, x, y$, and $z$, respectively, and $N$ is a nonlinear operator. Applying the fuzzy variational iteration method, we can express the following 
correction functional (in $t$-, $x$-, $y$-, and $z$-directions), respectively, as

$$
\left.\begin{array}{l}
\underline{u}_{m+1}(x, y, z, t ; r)=\underline{u}_{m}(x, y, z, t ; r)+\int_{0}^{t} \lambda_{1}\left[L_{s} \underline{u}_{m}+\left(L_{x}+L_{y}+L_{z}+N\right) \underline{u}_{m}-g\right] d s, \\
\underline{u}_{m+1}(x, y, z, t ; r)=\underline{u}_{m}(x, y, z, t ; r)+\int_{0}^{x} \lambda_{2}\left[L_{s} \underline{u}_{m}+\left(L_{t}+L_{y}+L_{z}+N\right) \underline{u}_{m}-g\right] d s, \\
\underline{u}_{m+1}(x, y, z, t ; r)=\underline{u}_{m}(x, y, z, t ; r)+\int_{0}^{y} \lambda_{3}\left[L_{s} \underline{u}_{m}+\left(L_{x}+L_{t}+L_{z}+N\right) \underline{u}_{m}-g\right] d s, \\
\underline{u}_{m+1}(x, y, z, t ; r)=\underline{u}_{m}(x, y, z, t ; r)+\int_{0}^{z} \lambda_{4}\left[L_{s} \underline{u}_{m}+\left(L_{x}+L_{y}+L_{t}+N\right) \underline{u}_{m}-g\right] d s,
\end{array}\right\}
$$

and

$$
\left.\begin{array}{l}
\bar{u}_{m+1}(x, y, z, t ; r)=\bar{u}_{m}(x, y, z, t ; r)+\int_{0}^{t} \lambda_{1}\left[L_{s} \bar{u}_{m}+\left(L_{x}+L_{y}+L_{z}+N\right) \bar{u}_{m}-g\right] d s, \\
\bar{u}_{m+1}(x, y, z, t ; r)=\bar{u}_{m}(x, y, z, t ; r)+\int_{0}^{x} \lambda_{2}\left[L_{s} \bar{u}_{m}+\left(L_{t}+L_{y}+L_{z}+N\right) \bar{u}_{m}-g\right] d s, \\
\bar{u}_{m+1}(x, y, z, t ; r)=\bar{u}_{m}(x, y, z, t ; r)+\int_{0}^{y} \lambda_{3}\left[L_{s} \bar{u}_{m}+\left(L_{x}+L_{t}+L_{z}+N\right) \bar{u}_{m}-g\right] d s, \\
\bar{u}_{m+1}(x, y, z, t ; r)=\bar{u}_{m}(x, y, z, t ; r)+\int_{0}^{z} \lambda_{4}\left[L_{s} \bar{u}_{m}+\left(L_{x}+L_{y}+L_{t}+N\right) \bar{u}_{m}-g\right] d s,
\end{array}\right\}
$$

where $\lambda_{1}, \lambda_{2}, \lambda_{3}$, and $\lambda_{4}$ are general Lagrange multipliers, which can specified optimally via the fuzzy variational theory, and $\tilde{u}_{m}(x, y, z, t ; r)=\left[\underline{u}_{m}(x, y, z, t ; r), \bar{u}_{m}(x, y, z, t ; r)\right]$ via restricted variation with $\delta \underline{u}_{m}=0$ and $\delta \bar{u}_{m}=0$. Using the fuzzy variational iteration method, we first determine the Lagrange multipliers $\lambda_{i}, i=1,2,3,4$. We will easily obtain sequential approximations $\delta \tilde{u}_{m+1}, m \geq 0$, from solution $\tilde{u}(x, y, z, t ; r)=[\underline{u}(x, y, z, t ; r), \bar{u}(x, y, z, t ; r)]$, that is, a suitable choice for an experimental function that has an ambiguous value

$$
\tilde{u}_{0}(x, y, z, t ; r)=\left[\underline{u}_{0}(x, y, z, t ; r), \bar{u}_{0}(x, y, z, t ; r)\right] .
$$

Then the solution is given as follows:

$$
\begin{aligned}
& \underline{u}(x, y, z, t ; r)=\lim _{m \rightarrow \infty} \underline{u}_{m}(x, y, z, t ; r), \\
& \bar{u}(x, y, z, t ; r)=\lim _{m \rightarrow \infty} \bar{u}_{m}(x, y, z, t ; r) .
\end{aligned}
$$

\subsubsection{Fuzzy heat-like equations}

We consider the fuzzy heat-like equation of the form

$$
\tilde{u}_{t}=g_{1}(x, y, z) \odot \tilde{u}_{\mathrm{xx}} \oplus g_{2}(x, y, z) \odot \tilde{u}_{\mathrm{yy}} \oplus g_{3}(x, y, z) \odot \tilde{u}_{\mathrm{zz}}
$$

subject to the initial condition

$$
\tilde{u}(x, y, z, 0)=\tilde{g}_{4}(x, y, z) .
$$

The parametric form of (3.51) is

$$
\underline{u}_{t}=g_{1}(x, y, z) \underline{u}_{\mathrm{Xx}}+g_{2}(x, y, z) \underline{u}_{\mathrm{yy}}+g_{3}(x, y, z) \underline{u}_{\mathrm{zz}},
$$




$$
\bar{u}_{t}=g_{1}(x, y, z) \bar{u}_{\mathrm{xx}}+g_{2}(x, y, z) \bar{u}_{\mathrm{yy}}+g_{3}(x, y, z) \bar{u}_{\mathrm{zz}} .
$$

We construct a correction functional for equations (3.53) and (3.54) applying the fuzzy variational iteration method of the form

$$
\begin{aligned}
\underline{u}_{m+1}(x, y, z, t ; r)= & \underline{u}_{m}(x, y, z, t ; r)+\int_{0}^{t} \lambda\left[\left(\underline{u}_{m}\right)_{s}+g_{1}(x, y, z)\left(\underline{u}_{m}\right)_{\mathrm{xx}}+g_{2}(x, y, z)\left(\underline{u}_{m}\right)_{\mathrm{yy}}\right. \\
& \left.+g_{3}(x, y, z)\left(\underline{u}_{m}\right)_{\mathrm{zz}}\right] d s \\
\bar{u}_{m+1}(x, y, z, t ; r)= & \bar{u}_{m}(x, y, z, t ; r)+\int_{0}^{t} \lambda\left[\left(\bar{u}_{m}\right)_{s}+g_{1}(x, y, z)\left(\bar{u}_{m}\right)_{\mathrm{xx}}+g_{2}(x, y, z)\left(\bar{u}_{m}\right)_{\mathrm{yy}}\right. \\
& \left.+g_{3}(x, y, z)\left(\bar{u}_{m}\right)_{\mathrm{zz}}\right] d s
\end{aligned}
$$

where $m \geq 0$, and

$$
\begin{aligned}
& \underline{u}_{0}(x, y, z, t ; r)=\underline{u}(x, y, z, 0 ; r), \\
& \bar{u}_{0}(x, y, z, t ; r)=\bar{u}(x, y, z, 0 ; r) .
\end{aligned}
$$

The fuzzy variational iteration method solution $\tilde{u}(x, y, z, t ; r)=[\underline{u}(x, y, z, t ; r), \bar{u}(x, y, z, t ; r)]$ takes the form

$$
\begin{aligned}
& \underline{u}(x, y, z, t ; r)=\lim _{m \rightarrow \infty} \underline{u}_{m}(x, y, z, t ; r), \\
& \bar{u}(x, y, z, t ; r)=\lim _{m \rightarrow \infty} \bar{u}_{m}(x, y, z, t ; r),
\end{aligned}
$$

where $\tilde{u}_{m}(x, y, z, t ; r)=\left[\underline{u}_{m}(x, y, z, t ; r), \bar{u}_{m}(x, y, z, t ; r)\right]$ will be recursively specified.

\subsubsection{Inhomogeneous fuzzy heat-like equations}

We consider the inhomogeneous fuzzy heat-like equation of the form

$$
\tilde{u}_{t}=\tilde{\xi}(x, y, z) \oplus g_{1}(x, y, z) \odot \tilde{u}_{\mathrm{xx}} \oplus g_{2}(x, y, z) \odot \tilde{u}_{\mathrm{yy}} \oplus g_{3}(x, y, z) \odot \tilde{u}_{\mathrm{zz}}
$$

subject to the initial condition

$$
\tilde{u}(x, y, z, 0)=\tilde{g}_{4}(x, y, z) \text {. }
$$

The parametric form of (3.57) is

$$
\begin{aligned}
& \underline{u}_{t}=\underline{\xi}(x, y, z)+g_{1}(x, y, z) \underline{u}_{\mathrm{xx}}+g_{2}(x, y, z) \underline{u}_{\mathrm{yy}}+g_{3}(x, y, z) \underline{u}_{\mathrm{zz}}, \\
& \bar{u}_{t}=\bar{\xi}(x, y, z)+g_{1}(x, y, z) \bar{u}_{\mathrm{xx}}+g_{2}(x, y, z) \bar{u}_{\mathrm{yy}}+g_{3}(x, y, z) \bar{u}_{\mathrm{zz}} .
\end{aligned}
$$

We construct a correction functional for equations (3.59) and (3.60) applying the variational iteration method of the form

$$
\underline{u}_{m+1}(x, y, z, t ; r)=\underline{u}_{m}(x, y, z, t ; r)+\int_{0}^{t} \lambda\left[\left(\underline{u}_{m}(x, y, z, t ; r)\right)_{s}-\underline{\xi}(x, y, z)-\underline{F}\right] d s,
$$




$$
\bar{u}_{m+1}(x, y, z, t ; r)=\bar{u}_{m}(x, y, z, t ; r)+\int_{0}^{t} \lambda\left[\left(\bar{u}_{m}(x, y, z, t ; r)\right)_{s}-\bar{\xi}(x, y, z)-\bar{F}\right] d s
$$

where $m \geq 0$,

$$
\begin{aligned}
& \underline{u}_{0}(x, y, z, t ; r)=\underline{u}(x, y, z, 0 ; r), \\
& \bar{u}_{0}(x, y, z, t ; r)=\bar{u}(x, y, z, 0 ; r),
\end{aligned}
$$

and the above-mentioned $\tilde{\xi}(x, y, z ; r)=[\underline{\xi}(x, y, z ; r), \bar{\xi}(x, y, z ; r)]$ is the fuzzy inhomogeneous term; however, $\underline{F}$ and $\bar{F}$ are

$$
\begin{aligned}
& \underline{F}=g_{1}\left(\underline{u}_{m}(x, y, z, t ; r)\right)_{\mathrm{xx}}+g_{2}\left(\underline{u}_{m}(x, y, z, t ; r)\right)_{\mathrm{yy}}+g_{3}\left(\underline{u}_{m}(x, y, z, t ; r)\right)_{\mathrm{zz}}, \\
& \bar{F}=g_{1}\left(\bar{u}_{m}(x, y, z, t ; r)\right)_{\mathrm{xx}}+g_{2}\left(\bar{u}_{m}(x, y, z, t ; r)\right)_{\mathrm{yy}}+g_{3}\left(\bar{u}_{m}(x, y, z, t ; r)\right)_{\mathrm{zz}} .
\end{aligned}
$$

The fuzzy variational iteration method solution $\tilde{u}(x, y, z, t ; r)=[\underline{u}(x, y, z, t ; r), \bar{u}(x, y, z, t ; r)]$ takes the form

$$
\begin{aligned}
& \underline{u}(x, y, z, t ; r)=\lim _{m \rightarrow \infty}=\underline{u}_{m}(x, y, z, t ; r), \\
& \bar{u}(x, y, z, t ; r)=\lim _{m \rightarrow \infty}=\bar{u}_{m}(x, y, z, t ; r),
\end{aligned}
$$

where $\tilde{u}_{m}(x, y, z, t ; r)=\left[\underline{u}_{m}(x, y, z, t ; r), \bar{u}_{m}(x, y, z, t ; r)\right]$ will be recursively specified.

\subsubsection{Fuzzy wave-like equations}

We consider the fuzzy wave-like equation of the form

$$
\tilde{u}_{t t}=h_{1}(x, y, z) \odot \tilde{u}_{\mathrm{xx}} \oplus h_{2}(x, y, z) \odot \tilde{u}_{\mathrm{yy}} \oplus h_{3}(x, y, z) \odot \tilde{u}_{\mathrm{zz}}
$$

subject to the initial condition

$$
\tilde{u}(x, y, z, 0)=\tilde{h}_{4}(x, y, z), \quad \tilde{u}_{t}(x, y, z, 0)=\tilde{h}_{5}(x, y, z) .
$$

The parametric form of (3.65) is

$$
\begin{aligned}
& \underline{u}_{t t}=h_{1}(x, y, z) \underline{u}_{\mathrm{xx}}+h_{2}(x, y, z) \underline{u}_{\mathrm{yy}}+h_{3}(x, y, z) \underline{u}_{\mathrm{zz}} \\
& \bar{u}_{t t}=h_{1}(x, y, z) \bar{u}_{\mathrm{xx}}+h_{2}(x, y, z) \bar{u}_{\mathrm{yy}}+h_{3}(x, y, z) \bar{u}_{\mathrm{zz}} .
\end{aligned}
$$

According to the variational iteration method, equations (3.67) and (3.68) take the form

$$
\begin{aligned}
\underline{u}_{m+1}(x, y, z, t ; r)= & \underline{u}_{m}(x, y, z, t ; r)+\int_{0}^{t} \lambda\left[\left(\underline{u}_{m}\right)_{\mathrm{ss}}+h_{1}(x, y, z)\left(\underline{u}_{m}\right)_{\mathrm{xx}}+h_{2}(x, y, z)\left(\underline{u}_{m}\right)_{\mathrm{yy}}\right. \\
& \left.+h_{3}(x, y, z)\left(\underline{u}_{m}\right)_{\mathrm{zz}}\right] d s \\
\bar{u}_{m+1}(x, y, z, t ; r)= & \bar{u}_{m}(x, y, z, t ; r)+\int_{0}^{t} \lambda\left[\left(\bar{u}_{m}\right)_{\mathrm{ss}}+h_{1}(x, y, z)\left(\bar{u}_{m}\right)_{\mathrm{xx}}+h_{2}(x, y, z)\left(\bar{u}_{m}\right)_{\mathrm{yy}}\right. \\
& \left.+h_{3}(x, y, z)\left(\bar{u}_{m}\right)_{z z}\right] d s,
\end{aligned}
$$


where $m \geq 0$, and $\tilde{u}_{0}(x, y, z, t ; r)=\left[\underline{u}(x, y, z, 0 ; r)+t \underline{u}_{t}(x, y, z, 0 ; r), \bar{u}(x, y, z, 0 ; r)+t \bar{u}_{t}(x, y, z\right.$, $0 ; r)]$.

The fuzzy variational iteration method solution $\tilde{u}(x, y, z, t ; r)=[\underline{u}(x, y, z, t ; r), \bar{u}(x, y, z, t ; r)]$ can be expressed as

$$
\begin{aligned}
& \underline{u}(x, y, z, t ; r)=\lim _{m \rightarrow \infty} \underline{u}_{m}(x, y, z, t ; r), \\
& \bar{u}(x, y, z, t ; r)=\lim _{m \rightarrow \infty} \bar{u}_{m}(x, y, z, t ; r),
\end{aligned}
$$

where $\tilde{u}_{m}(x, y, z, t ; r)=\left[\underline{u}_{m}(x, y, z, t ; r), \bar{u}_{m}(x, y, z, t ; r)\right]$ will be recursively specified.

\section{Examples}

In this section, we demonstrate how fuzzy Adomian decomposition method and fuzzy variational iteration method can be easily applied to get the exact solutions of the fuzzy heat-like and wave-like equations with variable coefficients.

Example 4.1 We consider the following one-dimensional fuzzy heat-like equation with variable coefficients:

$$
\tilde{u}_{t}=\frac{1}{2} x^{2} \odot \tilde{u}_{\mathrm{xx}}, \quad 0<x<1, t>0,
$$

with the boundary conditions

$$
\tilde{u}(0, t)=0, \quad \tilde{u}(1, t)=\exp (t)
$$

and the initial condition

$$
\tilde{u}(x, 0)=\left[(-0.5+1.5 r)^{n},(1.5-0.5 r)^{n}\right] \odot x^{2},
$$

where $(n=1,2,3, \ldots)$.

The parametric form of (4.1) is

$$
\begin{array}{ll}
\underline{u}_{t}=\frac{1}{2} x^{2} \underline{u}_{x x}, & 0<x<1, t>0, \\
\bar{u}_{t}=\frac{1}{2} x^{2} \bar{u}_{x x}, & 0<x<1, t>0,
\end{array}
$$

for $r \in[0,1]$, where $\underline{u}$ stands for $\underline{u}(x, t ; r)$, and similarly $\bar{u}$.

Case [A]. Fuzzy Adomian decomposition method

In an operator form, equations (4.4) and (4.5) are

$$
\begin{array}{ll}
L_{t} \underline{u}=\frac{1}{2} x^{2} \underline{u}_{\mathrm{xx}}, & 0<x<1, \\
L_{t} \bar{u}=\frac{1}{2} x^{2} \bar{u}_{\mathrm{xx}}, & 0<x<1 .
\end{array}
$$

Using the initial condition (4.3) and applying $L_{t}^{-1}$ to equations (4.6) and (4.7), we get

$$
\underline{u}(x, t ; r)=(1.5 r-0.5)^{n} x^{2}+L_{t}^{-1}\left[\frac{1}{2} x^{2} u_{\mathrm{xx}}\right]
$$




$$
\bar{u}(x, t ; r)=(1.5-0.5 r)^{n} x^{2}+L_{t}^{-1}\left[\frac{1}{2} x^{2} u_{\mathrm{xx}}\right]
$$

Substituting the ADM series (3.15) and (3.16) for $\tilde{u}$ into (4.8) and (4.9), we define

$$
\begin{aligned}
& \sum_{m=0}^{\infty} \underline{u}_{m}(x, t ; r)=(1.5 r-0.5)^{n} x^{2}+L_{t}^{-1}\left[\frac{1}{2} x^{2}\left(\sum_{m=0}^{\infty} \underline{u}_{m}(x, t ; r)\right)_{\mathrm{xx}}\right] \\
& \sum_{m=0}^{\infty} \bar{u}_{m}(x, t ; r)=(1.5-0.5 r)^{n} x^{2}+L_{t}^{-1}\left[\frac{1}{2} x^{2}\left(\sum_{m=0}^{\infty} \bar{u}_{m}(x, t ; r)\right)_{\mathrm{xx}}\right] .
\end{aligned}
$$

Next, we use the recursive relations

$$
\left.\begin{array}{l}
\underline{u}_{0}(x, t ; r)=(1.5 r-0.5)^{n} x^{2}, \\
\underline{u}_{m+1}(x, t ; r)=L_{t}^{-1}\left[\frac{1}{2} x^{2}\left(\underline{u}_{m}\right)_{\mathrm{xx}}\right], \quad j \geq 0,
\end{array}\right\}
$$

and

$$
\left.\begin{array}{l}
\bar{u}_{0}(x, t ; r)=(1.5-0.5 r)^{n} x^{2} \\
\bar{u}_{m+1}(x, t ; r)=L_{t}^{-1}\left[\frac{1}{2} x^{2}\left(\bar{u}_{m}\right)_{\mathrm{xx}}\right], \quad j \geq 0 .
\end{array}\right\}
$$

This in turn gives the first few components:

$$
\left.\begin{array}{rl}
\underline{u}_{0}(x, t ; r) & =(1.5 r-0.5)^{n} x^{2}, \\
\underline{u}_{1}(x, t ; r) & =L_{t}^{-1}\left[\frac{1}{2} L^{-1} x^{2}\left(\underline{u}_{0}\right)_{\mathrm{xx}}\right]=(1.5 r-0.5)^{n} x^{2} t, \\
\underline{u}_{2}(x, t ; r) & =L_{t}^{-1}\left[\frac{1}{2} L^{-1} x^{2}\left(\underline{u}_{1}\right)_{\mathrm{xx}}\right]=(1.5 r-0.5)^{n} x^{2} \frac{t^{2}}{2 !}, \\
\underline{u}_{3}(x, t ; r) & =L_{t}^{-1}\left[\frac{1}{2} L^{-1} x^{2}\left(\underline{u}_{2}\right)_{\mathrm{xx}}\right]=(1.5 r-0.5)^{n} x^{2} \frac{t^{3}}{3 !}, \\
\underline{u}_{m}(x, t ; r) & =L_{t}^{-1}\left[\frac{1}{2} L^{-1} x^{2}\left(\underline{u}_{m}\right)_{\mathrm{xx}}\right]=(1.5 r-0.5)^{m} x^{2} \frac{t^{m}}{m !},
\end{array}\right\}
$$

and

$$
\left.\begin{array}{rl}
\bar{u}_{0}(x, t ; r) & =(1.5-0.5 r)^{n} x^{2}, \\
\bar{u}_{1}(x, t ; r) & =L_{t}^{-1}\left[\frac{1}{2} L^{-1} x^{2}\left(\bar{u}_{0}\right)_{\mathrm{xx}}\right]=(1.5-0.5 r)^{n} x^{2} t, \\
\bar{u}_{2}(x, t ; r) & =L_{t}^{-1}\left[\frac{1}{2} L^{-1} x^{2}\left(\bar{u}_{1}\right)_{\mathrm{xx}}\right]=(1.5-0.5 r)^{n} x^{2} \frac{t^{2}}{2 !}, \\
\bar{u}_{3}(x, t ; r) & =L_{t}^{-1}\left[\frac{1}{2} L^{-1} x^{2}\left(\bar{u}_{2}\right)_{\mathrm{xx}}\right]=(1.5-0.5 r)^{n} x^{2} \frac{t^{3}}{3 !}, \\
\vdots & \\
\bar{u}_{m}(x, t ; r) & =L_{t}^{-1}\left[\frac{1}{2} L^{-1} x^{2}\left(\bar{u}_{m}\right)_{\mathrm{xx}}\right]=(1.5-0.5 r)^{n} x^{2} \frac{t^{m}}{m !} \cdot
\end{array}\right\}
$$


Consequently, the solution for the standard fuzzy heat-like equation for $\alpha=1$ is given by

$$
\begin{aligned}
& \underline{u}(x, t ; r)=(1.5 r-0.5)^{n}\left[x^{2}\left(1+t+\frac{t^{2}}{2 !}+\frac{t^{3}}{3 !}+\cdots+\frac{t^{m}}{m !}\right)\right], \\
& \bar{u}(x, t ; r)=(1.5-0.5 r)^{n}\left[x^{2}\left(1+t+\frac{t^{2}}{2 !}+\frac{t^{3}}{3 !}+\cdots+\frac{t^{m}}{m !}\right)\right],
\end{aligned}
$$

and in the closed form by

$$
\tilde{u}(x, t ; r)=\left[(1.5 r-0.5)^{n},(1.5-0.5 r)^{n}\right] \odot\left(\exp (t) x^{2}\right), \quad 0 \leq r \leq 1 .
$$

\section{Case [B]. Fuzzy variational iteration method}

The fuzzy VIM schema of equations (4.4) and (4.5) are

$$
\begin{aligned}
& \underline{u}_{m+1}(x, t ; r)=\underline{u}_{m}(x, t ; r)+\int_{0}^{t} \lambda\left[\left(\underline{u}_{m}(x, s ; r)\right)_{\mathrm{s}}-\frac{x^{2}}{2}\left(\underline{u}_{m}(x, s ; r)\right)_{\mathrm{xx}}\right] d s, \\
& \bar{u}_{m+1}(x, t ; r)=\bar{u}_{m}(x, t ; r)+\int_{0}^{t} \lambda\left[\left(\bar{u}_{m}(x, s ; r)\right)_{\mathrm{s}}-\frac{x^{2}}{2}\left(\bar{u}_{m}(x, s ; r)\right)_{\mathrm{xx}}\right] d s .
\end{aligned}
$$

Making the above correction functional stationary, we have

$$
\begin{aligned}
& \delta \underline{u}_{m+1}(x, t ; r)=\delta \underline{u}_{m}(x, t ; r)+\delta \int_{0}^{t} \lambda\left[\left(\underline{u}_{m}(x, s ; r)\right)_{\mathrm{s}}-\frac{x^{2}}{2}\left(\underline{u}_{m}(x, s ; r)\right)_{\mathrm{xx}}\right] d s, \\
& \delta \bar{u}_{m+1}(x, t ; r)=\delta \bar{u}_{m}(x, t ; r)+\delta \int_{0}^{t} \lambda\left[\left(\bar{u}_{m}(x, s ; r)\right)_{\mathrm{s}}-\frac{x^{2}}{2}\left(\bar{u}_{m}(x, s ; r)\right)_{\mathrm{xx}}\right] d s .
\end{aligned}
$$

Therefore the stationary conditions are given by

$$
1+\left.\lambda\right|_{s=t}=0, \quad \lambda^{\prime}(s)=0 .
$$

By integrating the differential equation we have

$$
\lambda^{\prime}(s)=0 \quad \Rightarrow \quad \lambda(s)=c_{1} .
$$

Using the condition, we arrive at

$$
1+\left.\lambda\right|_{s=t}=0 \quad \Rightarrow \quad c_{1}=-1
$$

Therefore the Lagrange multiplier $\lambda$ is given by

$$
\lambda=-1 .
$$

Substituting the value of the Lagrange multiplier into equations (4.14) and (4.15) gives the following iterative formulas:

$$
\underline{u}_{m+1}(x, t ; r)=\underline{u}_{m}(x, t ; r)-\int_{0}^{t}\left[\left(\underline{u}_{m}(x, s ; r)\right)_{\mathrm{s}}-\frac{x^{2}}{2}\left(\underline{u}_{m}(x, s ; r)\right)_{\mathrm{xx}}\right] d s,
$$




$$
\bar{u}_{m+1}(x, t ; r)=\bar{u}_{m}(x, t ; r)-\int_{0}^{t}\left[\left(\bar{u}_{m}(x, s ; r)\right)_{\mathrm{s}}-\frac{x^{2}}{2}\left(\bar{u}_{m}(x, s ; r)\right)_{\mathrm{xx}}\right] d s .
$$

Using iteration formulas (4.18) and (4.19) with the initial approximation $\tilde{u}_{0}(x, t ; r)=$ $\tilde{u}(x, 0 ; r)=\left[(-0.5+1.5 r)^{n},(1.5-0.5 r)^{n}\right] \odot x^{2}$, we get the following successive approximations:

$$
\left.\begin{array}{rl}
\underline{u}_{1}(x, t ; r) & =(1.5 r-0.5)^{n}(1+t) x^{2}, \\
\underline{u}_{2}(x, t ; r) & =(1.5 r-0.5)^{n}\left(1+t+\frac{t^{2}}{2 !}\right) x^{2}, \\
\underline{u}_{3}(x, t ; r) & =(1.5 r-0.5)^{n}\left(1+t+\frac{t^{2}}{2 !}+\frac{t^{3}}{3 !}\right) x^{2}, \\
\vdots & \\
\underline{u}_{m}(x, t ; r) & =(1.5 r-0.5)^{n}\left(1+t+\frac{t^{2}}{2 !}+\frac{t^{3}}{3 !}+\cdots+\frac{t^{m}}{m !}\right) x^{2},
\end{array}\right\}
$$

and

$$
\left.\begin{array}{rl}
\bar{u}_{1}(x, t ; r) & =(1.5-0.5 r)^{n}(1+t) x^{2}, \\
\bar{u}_{2}(x, t ; r) & =(1.5-0.5 r)^{n}\left(1+t+\frac{t^{2}}{2 !}\right) x^{2}, \\
\bar{u}_{3}(x, t ; r) & =(1.5-0.5 r)^{n}\left(1+t+\frac{t^{2}}{2 !}+\frac{t^{3}}{3 !}\right) x^{2}, \\
\vdots & \\
\bar{u}_{m}(x, t ; r) & =(1.5-0.5 r)^{n}\left(1+t+\frac{t^{2}}{2 !}+\frac{t^{3}}{3 !}+\cdots+\frac{t^{m}}{m !}\right) x^{2} .
\end{array}\right\}
$$

Consequently, the exact solution is given by

$$
\begin{aligned}
& \underline{u}(x, t ; r)=(1.5 r-0.5)^{n} \lim _{m \rightarrow \infty}\left[x^{2}\left(1+t+\frac{t^{2}}{2 !}+\frac{t^{3}}{3 !}+\cdots \frac{t^{m}}{m !}\right)\right], \\
& \bar{u}(x, t ; r)=(1.5-0.5 r)^{n} \lim _{m \rightarrow \infty}\left[x^{2}\left(1+t+\frac{t^{2}}{2 !}+\frac{t^{3}}{3 !}+\cdots+\frac{t^{m}}{m !}\right)\right],
\end{aligned}
$$

and we can rewrite the solution as follows:

$$
\tilde{u}(x, t ; r)=\left[(1.5 r-0.5)^{n},(1.5-0.5 r)^{n}\right] \odot\left(\exp (t) x^{2}\right), \quad 0 \leq r \leq 1 .
$$

Example 4.2 We consider the following two-dimensional fuzzy heat-like equation with variable coefficients:

$$
\tilde{u}_{t}=\frac{1}{2}\left(y^{2} \odot \tilde{u}_{\mathrm{xx}} \oplus x^{2} \odot \tilde{u}_{\mathrm{yy}}\right), \quad 0<x, y<1, t>0,
$$


with the Neumann boundary conditions

$$
\begin{array}{ll}
\tilde{u}_{x}(0, y, t)=0, & \tilde{u}_{x}(1, y, t)=2 \sinh t, \\
\tilde{u}_{y}(x, 0, t)=0, & \tilde{u}_{y}(x, 1, t)=2 \operatorname{coth} t,
\end{array}
$$

and the initial condition

$$
\tilde{u}(x, y, 0)=\left[(1+2 r)^{n},(5-2 r)^{n}\right] \ominus_{g H} y^{2},
$$

where $(n=1,2,3, \ldots)$.

The parametric form of $(4.22)$ is

$$
\begin{array}{ll}
\underline{u}_{t}=\frac{1}{2}\left(y^{2} \underline{u}_{\mathrm{xx}}+x^{2} \underline{u}_{\mathrm{yy}}\right), & 0<x, y<1, t>0, \\
\bar{u}_{t}=\frac{1}{2}\left(y^{2} \bar{u}_{\mathrm{xx}}+x^{2} \bar{u}_{\mathrm{yy}}\right), & 0<x, y<1, t>0,
\end{array}
$$

for $r \in[0,1]$, where $\underline{u}$ stands for $\underline{u}(x, y, t ; r)$, and similarly for $\bar{u}$.

\section{Case [A]. Fuzzy Adomian decomposition method}

In an operator form, equations (4.25) and (4.26) are

$$
\begin{aligned}
& L_{t} \underline{u}=\frac{1}{2}\left(y^{2} \underline{u}_{\mathrm{xx}}+x^{2} \underline{u}_{\mathrm{yy}}\right), \\
& L_{t} \bar{u}=\frac{1}{2}\left(y^{2} \bar{u}_{\mathrm{xx}}+x^{2} \bar{u}_{\mathrm{yy}}\right) .
\end{aligned}
$$

Applying the inverse operator $L^{-1}$ to both sides of equations (4.27) and (4.28), we obtain

$$
\begin{aligned}
& \underline{u}(x, y, t ; r)=(1+2 r)^{n}-y^{2}+\frac{1}{2} L_{t}^{-1}\left(y^{2} \underline{u}_{\mathrm{xx}}+x^{2} \underline{u}_{\mathrm{yy}}\right), \\
& \bar{u}(x, y, t ; r)=(5-2 r)^{n}-y^{2}+\frac{1}{2} L_{t}^{-1}\left(y^{2} \bar{u}_{\mathrm{xx}}+x^{2} \bar{u}_{\mathrm{yy}}\right) .
\end{aligned}
$$

Using the fuzzy ADM series for $\tilde{u}(x, y, t ; r)=[\underline{u}(x, y, t ; r), \bar{u}(x, y, t ; r)]$, we get

$$
\begin{aligned}
\sum_{m=0}^{\infty} \underline{u}_{m}(x, y, t ; r)= & (1+2 r)^{n}-y^{2}+\frac{1}{2} L_{t}^{-1}\left[y^{2}\left(\sum_{m=0}^{\infty} \underline{u}_{m}(x, y, t ; r)\right)_{\mathrm{xx}}\right. \\
& \left.+x^{2}\left(\sum_{m=0}^{\infty} \underline{u}_{m}(x, y, t ; r)\right)_{\mathrm{yy}}\right], \\
\sum_{m=0}^{\infty} \bar{u}_{m}(x, y, t ; r)= & (5-2 r)^{n}-y^{2}+\frac{1}{2} L_{t}^{-1}\left[y^{2}\left(\sum_{m=0}^{\infty} \bar{u}_{m}(x, y, t ; r)\right)_{\mathrm{xx}}\right. \\
& \left.+x^{2}\left(\sum_{m=0}^{\infty} \bar{u}_{m}(x, y, t ; r)\right)_{\mathrm{yy}}\right],
\end{aligned}
$$

where $m \geq 0$. 
Now we use the recursive relations

$$
\left.\begin{array}{l}
\underline{u}_{0}(x, y, t ; r)=(1+2 r)^{n}-y^{2}, \\
\underline{u}_{m+1}(x, y, t ; r)=\frac{1}{2} L_{t}^{-1}\left(y^{2}\left(\underline{u}_{m}\right)_{\mathrm{xx}}+x^{2}\left(\underline{u}_{m}\right)_{\mathrm{yy}}\right), \quad m \geq 0,
\end{array}\right\}
$$

and

$$
\left.\begin{array}{l}
\bar{u}_{0}(x, y, t ; r)=(5-2 r)^{n}-y^{2}, \\
\bar{u}_{m+1}(x, y, t ; r)=\frac{1}{2} L_{t}^{-1}\left(y^{2}\left(\bar{u}_{m}\right)_{\mathrm{xx}}+x^{2}\left(\bar{u}_{m}\right)_{\mathrm{yy}}\right), \quad m \geq 0,
\end{array}\right\}
$$

and thus the first few components are

$$
\begin{aligned}
& \underline{u}_{0}(x, y, t ; r)=(1+2 r)^{n}-y^{2}, \\
& \underline{u}_{1}(x, y, t ; r)=\frac{1}{2} L_{t}^{-1}\left[y^{2}\left(\underline{u}_{0}\right)_{\mathrm{xx}}+x^{2}\left(\underline{u}_{0}\right)_{\mathrm{yy}}\right]=(1+2 r)^{n}-x^{2} t, \\
& \underline{u}_{2}(x, y, t ; r)=\frac{1}{2} L_{t}^{-1}\left[y^{2}\left(\underline{u}_{1}\right)_{\mathrm{xx}}+x^{2}\left(\underline{u}_{1}\right)_{\mathrm{yy}}\right]=(1+2 r)^{n}-y^{2} \frac{t^{2}}{2 !}, \\
& \underline{u}_{3}(x, y, t ; r)=\frac{1}{2} L_{t}^{-1}\left[y^{2}\left(\underline{u}_{2}\right)_{\mathrm{xx}}+x^{2}\left(\underline{u}_{2}\right)_{\mathrm{yy}}\right]=(1+2 r)^{n}-x^{2} \frac{t^{3}}{3 !}, \\
& \vdots
\end{aligned}
$$

and

$$
\begin{aligned}
& \bar{u}_{0}(x, y, t ; r)=(5-2 r)^{n}-y^{2}, \\
& \bar{u}_{1}(x, y, t ; r)=\frac{1}{2} L_{t}^{-1}\left[y^{2}\left(\bar{u}_{0}\right)_{\mathrm{xx}}+x^{2}\left(\bar{u}_{0}\right)_{y y}\right]=(5-2 r)^{n}-x^{2} t, \\
& \bar{u}_{2}(x, y, t ; r)=\frac{1}{2} L_{t}^{-1}\left[y^{2}\left(\bar{u}_{1}\right)_{\mathrm{xx}}+x^{2}\left(\bar{u}_{1}\right)_{y y}\right]=(5-2 r)^{n}-y^{2} \frac{t^{2}}{2 !}, \\
& \bar{u}_{3}(x, y, t ; r)=\frac{1}{2} L_{t}^{-1}\left[y^{2}\left(\bar{u}_{2}\right)_{\mathrm{xx}}+x^{2}\left(\bar{u}_{2}\right)_{y y}\right]=(5-2 r)^{n}-x^{2} \frac{t^{3}}{3 !}, \\
& \vdots
\end{aligned}
$$

Thus the solution in a series form is

$$
\begin{aligned}
& \underline{u}(x, y, t ; r)=(1+2 r)^{n}-\left[x^{2}\left(t+\frac{t^{3}}{3 !}+\frac{t^{5}}{5 !}+\cdots\right)+y^{2}\left(1+\frac{t^{2}}{2 !}+\frac{t^{4}}{4 !}+\cdots\right)\right], \\
& \bar{u}(x, y, t ; r)=(5-2 r)^{n}-\left[x^{2}\left(t+\frac{t^{3}}{3 !}+\frac{t^{5}}{5 !}+\cdots\right)+y^{2}\left(1+\frac{t^{2}}{2 !}+\frac{t^{4}}{4 !}+\cdots\right)\right],
\end{aligned}
$$

and then the exact solution is

$$
\tilde{u}(x, y, t ; r)=\left[(1+2 r)^{n},(5-2 r)^{n}\right] \ominus_{g H}\left(x^{2} \sinh t+y^{2} \cosh t\right), \quad 0 \leq r \leq 1 .
$$


The correction functionals for equations (4.25) and (4.26) are

$$
\begin{aligned}
\underline{u}_{m+1}(x, y, t ; r)= & \underline{u}_{m}(x, y, t ; r)+\int_{0}^{t} \lambda\left[\left(\underline{u}_{m}(x, y, s ; r)\right)_{s}-\frac{y^{2}}{2}\left(\underline{u}_{m}(x, y, s ; r)\right)_{\mathrm{xx}}\right. \\
& \left.-\frac{x^{2}}{2}\left(\underline{u}_{m}(x, y, s ; r)\right)_{\mathrm{yy}}\right] d s, \quad m \geq 0, \\
\bar{u}_{m+1}(x, y, t ; r)= & \bar{u}_{m}(x, y, s ; r)+\int_{0}^{t} \lambda\left[\left(\bar{u}_{m}(x, y, s ; r)\right)_{s}-\frac{y^{2}}{2}\left(\bar{u}_{m}(x, y, s ; r)\right)_{\mathrm{xx}}\right. \\
& \left.-\frac{x^{2}}{2}\left(\bar{u}_{m}(x, y, s ; r)\right)_{\mathrm{yy}}\right] d s, \quad m \geq 0 .
\end{aligned}
$$

Making the above correction functional stationary, we have

$$
\begin{aligned}
\delta \underline{u}_{m+1}(x, y, t ; r)= & \delta \underline{u}_{m}(x, y, t ; r)+\delta \int_{0}^{t} \lambda\left[\left(\underline{u}_{m}(x, y, s ; r)\right)_{s}-\frac{y^{2}}{2}\left(\underline{u}_{m}(x, y, s ; r)\right)_{\mathrm{xx}}\right. \\
& \left.-\frac{x^{2}}{2}\left(\underline{u}_{m}(x, y, s ; r)\right)_{\mathrm{yy}}\right] d s, \quad m \geq 0, \\
\delta \bar{u}_{m+1}(x, y, t ; r)= & \delta \bar{u}_{m}(x, y, s ; r)+\delta \int_{0}^{t} \lambda\left[\left(\bar{u}_{m}(x, y, s ; r)\right)_{s}-\frac{y^{2}}{2}\left(\bar{u}_{m}(x, y, s ; r)\right)_{\mathrm{xx}}\right. \\
& \left.-\frac{x^{2}}{2}\left(\bar{u}_{m}(x, y, s ; r)\right)_{\mathrm{yy}}\right] d s, \quad m \geq 0 .
\end{aligned}
$$

Similarly, the stationary conditions are given by

$$
1+\left.\lambda\right|_{s=t}, \quad \lambda^{\prime}(s)=0
$$

and thus the Lagrange multiplier

$$
\lambda=-1
$$

Substituting the value of the Lagrange multiplier into equations (4.35) and (4.36) gives the iteration formulas

$$
\begin{aligned}
\underline{u}_{m+1}(x, y, t ; r)= & \underline{u}_{m}(x, y, t ; r)-\int_{0}^{t}\left[\left(\underline{u}_{m}(x, y, s ; r)\right)_{s}-\frac{y^{2}}{2}\left(\underline{u}_{m}(x, y, s ; r)\right)_{\mathrm{xx}}\right. \\
& \left.-\frac{x^{2}}{2}\left(\underline{u}_{m}(x, y, s ; r)\right)_{\mathrm{yy}}\right] d s, \\
\bar{u}_{m+1}(x, y, t ; r)= & \bar{u}_{m}(x, y, s ; r)-\int_{0}^{t}\left[\left(\bar{u}_{m}(x, y, s ; r)\right)_{s}-\frac{y^{2}}{2}\left(\bar{u}_{m}(x, y, s ; r)\right)_{\mathrm{xx}}\right. \\
& \left.-\frac{x^{2}}{2}\left(\bar{u}_{m}(x, y, s ; r)\right)_{\mathrm{yy}}\right] d s .
\end{aligned}
$$

Using the iteration formulas of equations (4.39) and (4.40) with the initial conditions $\tilde{u}_{0}(x, y, t)=\tilde{u}(x, y, t)=\left[(1+2 r)^{n},(5-2 r)^{n}\right] \ominus_{g H} y^{2}, n=1,2,3, \ldots$, we get the following suc- 
cessive approximations:

$$
\left.\begin{array}{rl}
\underline{u}_{1}(x, y, t ; r) & =(1+2 r)^{n}-\left(t x^{2}+y^{2}\right), \\
\underline{u}_{2}(x, y, t ; r)= & (1+2 r)^{n}-\left[t x^{2}+\left(1+\frac{t^{2}}{2 !}\right) y^{2}\right] \\
\underline{u}_{3}(x, y, t ; r)= & (1+2 r)^{n}-\left[\left(t+\frac{t^{3}}{3 !}\right) x^{2}+\left(1+\frac{t^{2}}{2 !}\right) y^{2}\right], \\
\vdots & \\
\underline{u}_{m}(x, y, t ; r)= & (1+2 r)^{n}-\left[\left(t+\frac{t^{3}}{3 !}+\cdots+\frac{t^{2 m+1}}{(2 m+1) !}\right) x^{2}\right. \\
& \left.+\left(1+\frac{t^{2}}{2 !}+\cdots+\frac{t^{2 m}}{(2 m) !}\right) y^{2}\right]
\end{array}\right\}
$$

and

$$
\begin{aligned}
\bar{u}_{1}(x, y, t ; r)= & (5-2 r)^{n}-\left(t x^{2}+y^{2}\right) \\
\bar{u}_{2}(x, y, t ; r)= & (5-2 r)^{n}-\left[\left(t x^{2}+\left(1+\frac{t^{2}}{2 !}\right) y^{2}\right)\right], \\
\bar{u}_{3}(x, y, t ; r)= & (5-2 r)^{n}-\left[\left(t+\frac{t^{3}}{3 !}\right) x^{2}+\left(1+\frac{t^{2}}{2 !}\right) y^{2}\right], \\
\vdots & \\
\bar{u}_{m}(x, y, t ; r)= & (5-2 r)^{n}-\left[\left(t+\frac{t^{3}}{3 !}+\cdots+\frac{t^{2 m+1}}{(2 m+1) !}\right) x^{2}\right. \\
& \left.+\left(1+\frac{t^{2}}{2 !}+\cdots+\frac{t^{2 m}}{(2 m) !}\right) y^{2}\right] .
\end{aligned}
$$

Consequently, the exact solution is given by

$$
\begin{aligned}
\underline{u}(x, y, t ; r)= & (1+2 r)^{n}-\left[\lim _{m \rightarrow \infty}\left(t+\frac{t^{3}}{3 !}+\cdots+\frac{t^{2 m+1}}{(2 m+1) !}\right) x^{2}\right. \\
& \left.+\left(1+\frac{t^{2}}{2 !}+\cdots+\frac{t^{2 m}}{(2 m) !}\right) y^{2}\right], \\
\bar{u}(x, y, t ; r)= & (5-2 r)^{n}-\left[\lim _{m \rightarrow \infty}\left(t+\frac{t^{3}}{3 !}+\cdots+\frac{t^{2 m+1}}{(2 m+1) !}\right) x^{2}\right. \\
& \left.+\left(1+\frac{t^{2}}{2 !}+\cdots+\frac{t^{2 m}}{(2 m) !}\right) y^{2}\right],
\end{aligned}
$$

and we can rewrite the solution as follows:

$$
\tilde{u}(x, y, t ; r)=\left[(1+2 r)^{n},(5-2 r)^{n}\right] \ominus_{g H}\left(x^{2} \sinh t+y^{2} \cosh t\right), \quad 0 \leq r \leq 1 .
$$


Example 4.3 We consider the following three-dimensional inhomogeneous fuzzy heatlike equation with variable coefficients:

$$
\tilde{u}_{t}=\tilde{\tau}(x, y, z) \oplus \frac{1}{36}\left[x^{2} \odot \tilde{u}_{\mathrm{xx}} \oplus y^{2} \odot \tilde{u}_{\mathrm{yy}} \oplus z^{2} \odot \tilde{u}_{\mathrm{zz}}\right], \quad 0<x, y, z<1, t>0,
$$

with the boundary conditions

$$
\begin{array}{ll}
\tilde{u}(0, y, z, t)=0, & \tilde{u}(1, y, z, t)=y^{4} z^{4}(\exp (t)-1), \\
\tilde{u}(x, 0, z, t)=0, & \tilde{u}(x, 1, z, t)=x^{4} z^{4}(\exp (t)-1), \\
\tilde{u}(x, y, 0, t)=0, & \tilde{u}(x, y, 1, t)=x^{4} y^{4}(\exp (t)-1),
\end{array}
$$

and the initial condition

$$
\tilde{u}(x, y, z, 0)=\tilde{0},
$$

where

$$
\begin{aligned}
\tilde{\tau}(x, y, z ; r) & =(-1,0,1)^{n} \odot(x y z)^{4}, \\
& =\left[(r-1)^{n},(1-r)^{n}\right] \odot(x y z)^{4}, \quad 0 \leq r \leq 1(n=1,2,3, \ldots), \tilde{0} \in E^{1} .
\end{aligned}
$$

The parametric form of (4.43) is

$$
\begin{array}{ll}
\underline{u}_{t}=(r-1)^{n}(x y z)^{4}+\frac{1}{36}\left[x^{2} \underline{u}_{\mathrm{xx}}+y^{2} \underline{u}_{\mathrm{yy}}+z^{2} \underline{u}_{\mathrm{zz}}\right], & 0<x, y, z<1, t>0, \\
\bar{u}_{t}=(1-r)^{n}(x y z)^{4}+\frac{1}{36}\left[x^{2} \bar{u}_{\mathrm{xx}}+y^{2} \bar{u}_{\mathrm{yy}}+z^{2} \bar{u}_{\mathrm{zz}}\right], & 0<x, y, z<1, t>0,
\end{array}
$$

for $r \in[0,1]$, where $\underline{u}$ stands for $\underline{u}(x, y, z, t ; r)$, and similarly for $\bar{u}$.

\section{Case [A]. Fuzzy Adomian decomposition method}

Working equations (4.46) and (4.47) are

$$
\begin{aligned}
& L_{t} \underline{u}=(r-1)^{n} x^{4} y^{4} z^{4}+\frac{1}{36}\left[x^{2} \underline{u}_{\mathrm{xx}}+y^{2} \underline{u}_{\mathrm{yy}}+y^{2} \underline{u}_{\mathrm{zz}}\right](r), \\
& L_{t} \bar{u}=(1-r)^{n} x^{4} y^{4} z^{4}+\frac{1}{36}\left[x^{2} \bar{u}_{\mathrm{xx}}+y^{2} \bar{u}_{\mathrm{yy}}+z^{2} \bar{u}_{\mathrm{zz}}\right](r) .
\end{aligned}
$$

Applying $L^{-1}$ to equations (4.48) and (4.49), we obtain

$$
\begin{aligned}
& \underline{u}(x, y, z, t ; r)=(r-1)^{n} x^{4} y^{4} z^{4} t+\frac{1}{36} L_{t}^{-1}\left[x^{2} \underline{u}_{\mathrm{xx}}+y^{2} \underline{u}_{\mathrm{yy}}+z^{2} \underline{u}_{\mathrm{zz}}\right](r), \\
& \bar{u}(x, y, z, t ; r)=(1-r)^{n} x^{4} y^{4} z^{4} t+\frac{1}{36} L_{t}^{-1}\left[x^{2} \bar{u}_{\mathrm{xx}}+y^{2} \bar{u}_{\mathrm{yy}}+z^{2} \bar{u}_{\mathrm{zz}}\right](r) .
\end{aligned}
$$


Using the fuzzy ADM series (3.27) and (3.28) for the solution $\tilde{u}(x, y, z, t ; r)=[\underline{u}(x, y, z, t ; r)$, $\bar{u}(x, y, z, t ; r)]$ gives

$$
\begin{aligned}
\sum_{n=0}^{\infty} \underline{u}_{m}(x, y, z, t ; r)= & (r-1)^{n} x^{4} y^{4} z^{4} t+\frac{1}{36}\left[x^{2}\left(\sum_{m=0}^{\infty} \underline{u}_{m}(x, y, z, t ; r)\right)_{\mathrm{xx}}\right. \\
& \left.+y^{2}\left(\sum_{m=0}^{\infty} \underline{u}_{m}(x, y, z, t ; r)\right)_{\mathrm{yy}}+z^{2}\left(\sum_{m=0}^{\infty} \underline{u}_{m}(x, y, z, t ; r)\right)_{\mathrm{zz}}\right], \\
\sum_{m=0}^{\infty} \bar{u}_{m}(x, y, z, t ; r)= & (1-r)^{n} x^{4} y^{4} z^{4} t+\frac{1}{36}\left[x^{2}\left(\sum_{m=0}^{\infty} \bar{u}_{m}(x, y, z, t ; r)\right)_{\mathrm{xx}}\right. \\
& \left.+y^{2}\left(\sum_{m=0}^{\infty} \bar{u}_{m}(x, y, z, t ; r)\right)_{\mathrm{yy}}+z^{2}\left(\sum_{m=0}^{\infty} \bar{u}_{m}(x, y, z, t ; r)\right)_{\mathrm{zz}}\right] .
\end{aligned}
$$

We use the recursive relation

$$
\begin{aligned}
& \underline{u}_{0}(x, y, z, t ; r)=(r-1)^{n} x^{4} y^{4} z^{4} t, \\
& \underline{u}_{m+1}(x, y, z, t ; r)=\frac{1}{36} L_{t}^{-1}\left[x^{2}\left(\sum_{m=0}^{\infty} \underline{u}_{m}(x, y, z, t ; r)\right)_{\mathrm{xx}}+y^{2}\left(\sum_{m=0}^{\infty} \underline{u}_{m}(x, y, z, t ; r)\right)_{\mathrm{yy}}\right. \\
& \left.+z^{2}\left(\sum_{m=0}^{\infty} \underline{u}_{m}(x, y, z, t ; r)\right)_{\mathrm{zz}}\right], \quad m \geq 0,
\end{aligned}
$$

and

$$
\begin{aligned}
\bar{u}_{0}(x, y, z, t ; r)=(1-r)^{n} x^{4} y^{4} z^{4} t & \\
\bar{u}_{m+1}(x, y, z, t ; r)= & \frac{1}{36} L_{t}^{-1}\left[x^{2}\left(\sum_{m=0}^{\infty} \bar{u}_{m}(x, y, t ; r)\right)_{\mathrm{xx}}+y^{2}\left(\sum_{m=0}^{\infty} \bar{u}_{m}(x, y, z, t ; r)\right)_{\mathrm{yy}}\right. \\
& \left.+z^{2}\left(\sum_{m=0}^{\infty} \bar{u}_{m}(x, y, z, t ; r)\right)_{\mathrm{zz}}\right], \quad m \geq 0 .
\end{aligned}
$$

The first few components of $\tilde{u}(x, y, z, t ; r)=[\underline{u}(x, y, z, t ; r), \bar{u}(x, y, z, t ; r)]$ are

$$
\left.\begin{array}{rl}
\underline{u}_{0}(x, y, z, t ; r) & =(r-1)^{n} x^{4} y^{4} z^{4} t, \\
\underline{u}_{1}(x, y, z, t ; r) & =\frac{1}{36} L_{t}^{-1}\left[x^{2}\left(\underline{u}_{0}\right)_{\mathrm{xx}}+y^{2}\left(\underline{u}_{0}\right)_{\mathrm{yy}}+z^{2}\left(\underline{u}_{0}\right)_{\mathrm{zz}}\right]=(r-1)^{n} x^{4} y^{4} z^{4} \frac{t^{2}}{2 !}, \\
\underline{u}_{2}(x, y, z, t ; r) & =\frac{1}{36} L_{t}^{-1}\left[x^{2}\left(\underline{u}_{1}\right)_{\mathrm{xx}}+y^{2}\left(\underline{u}_{1}\right)_{\mathrm{yy}}+z^{2}\left(\underline{u}_{1}\right)_{\mathrm{zz}}\right]=(r-1)^{n} x^{4} y^{4} z^{4} \frac{t^{3}}{3 !}, \\
\underline{u}_{3}(x, y, z, t ; r) & =\frac{1}{36} L_{t}^{-1}\left[x^{2}\left(\underline{u}_{2}\right)_{\mathrm{xx}}+y^{2}\left(\underline{u}_{2}\right)_{\mathrm{yy}}+z^{2}\left(\underline{u}_{2}\right)_{\mathrm{zz}}\right]=(r-1)^{n} x^{4} y^{4} z^{4} \frac{t^{4}}{4 !}, \\
\vdots & \\
\underline{u}_{m}(x, y, z, t ; r) & =\frac{1}{36} L_{t}^{-1}\left[x^{2}\left(\underline{u}_{m}\right)_{\mathrm{xx}}+y^{2}\left(\underline{u}_{m}\right)_{\mathrm{yy}}+z^{2}\left(\underline{u}_{m}\right)_{\mathrm{zz}}\right]=(r-1)^{n} x^{4} y^{4} z^{4} \frac{t^{m}}{m !},
\end{array}\right\}
$$


and

$$
\left.\begin{array}{rl}
\bar{u}_{0}(x, y, z, t ; r) & =(1-r)^{n} x^{4} y^{4} z^{4} t, \\
\bar{u}_{1}(x, y, z, t ; r) & =\frac{1}{36} L_{t}^{-1}\left[x^{2}\left(\bar{u}_{0}\right)_{\mathrm{xx}}+y^{2}\left(\bar{u}_{0}\right)_{\mathrm{yy}}+z^{2}\left(\bar{u}_{0}\right)_{\mathrm{zz}}\right]=(1-r)^{n} x^{4} y^{4} z^{4} \frac{t^{2}}{2 !}, \\
\bar{u}_{2}(x, y, z, t ; r) & =\frac{1}{36} L_{t}^{-1}\left[x^{2}\left(\bar{u}_{1}\right)_{\mathrm{xx}}+y^{2}\left(\bar{u}_{1}\right)_{\mathrm{yy}}+z^{2}\left(\bar{u}_{1}\right)_{\mathrm{zz}}\right]=(1-r)^{n} x^{4} y^{4} z^{4} \frac{t^{3}}{3 !}, \\
\bar{u}_{3}(x, y, z, t ; r) & =\frac{1}{36} L_{t}^{-1}\left[x^{2}\left(\bar{u}_{2}\right)_{\mathrm{xx}}+y^{2}\left(\bar{u}_{2}\right)_{\mathrm{yy}}+z^{2}\left(\bar{u}_{2}\right)_{\mathrm{zz}}\right]=(1-r)^{n} x^{4} y^{4} z^{4} \frac{t^{4}}{4 !}, \\
\vdots & \\
\bar{u}_{m}(x, y, z, t ; r) & =\frac{1}{36} L_{t}^{-1}\left[x^{2}\left(\bar{u}_{m}\right)_{\mathrm{xx}}+y^{2}\left(\bar{u}_{m}\right)_{\mathrm{yy}}+z^{2}\left(\bar{u}_{m}\right)_{\mathrm{zz}}\right]=(1-r)^{n} x^{4} y^{4} z^{4} \frac{t^{m}}{m !} .
\end{array}\right\}
$$

The solution for the inhomogeneous fuzzy heat-like equations (4.46) and (4.47) in a series form is given by

$$
\begin{aligned}
& \underline{u}(x, y, z, t ; r)=(r-1)^{n} x^{4} y^{4} z^{4}\left[t+\frac{t^{2}}{2 !}+\frac{t^{3}}{3 !}+\frac{t^{4}}{4 !}+\cdots+\frac{t^{m}}{m !}\right], \\
& \bar{u}(x, y, z, t, r)=(1-r)^{n} x^{4} y^{4} z^{4}\left[t+\frac{t^{2}}{2 !}+\frac{t^{3}}{3 !}+\frac{t^{4}}{4 !}+\cdots+\frac{t^{m}}{m !}\right] .
\end{aligned}
$$

The exact solution of equations (4.46) and (4.47) when $\alpha=1$ is

$$
\tilde{u}(x, y, z, t ; r)=\left[(r-1)^{n},(1-r)^{n}\right] \odot\left(x^{4} y^{4} z^{4}(\exp (t)-1)\right), \quad 0 \leq r \leq 1 .
$$

\section{Case [B]. Fuzzy variational iteration method}

The correction functionals for equations (4.46) and (4.47) are

$$
\begin{aligned}
\underline{u}_{m+1}(x, y, z, t ; r)= & \underline{u}_{m}(x, y, z, t ; r) \\
& +\int_{0}^{t} \lambda\left[\left(\underline{u}_{m}(x, y, z, s ; r)\right)_{s}-(r-1)^{n}(x y z)^{4}-\underline{F}\right] d s, \\
\bar{u}_{m+1}(x, y, z, t ; r)= & \bar{u}_{m}(x, y, z, t ; r) \\
& +\int_{0}^{t} \lambda\left[\left(\bar{u}_{m}(x, y, z, s ; r)\right)_{s}-(1-r)^{n}(x y z)^{4}-\bar{F}\right] d s,
\end{aligned}
$$

where $m \geq 0$,

$$
\begin{aligned}
& \underline{F}=\frac{1}{36}\left(x^{2}\left(\underline{u}_{m}(x, y, z, s ; r)\right)_{\mathrm{xx}}+\left(y^{2}\left(\underline{u}_{m}(x, y, z, s ; r)\right)_{\mathrm{yy}}+\left(z^{2}\left(\underline{u}_{m}(x, y, z, s ; r)\right)_{\mathrm{zz}}\right),\right.\right. \\
& \bar{F}=\frac{1}{36}\left(x^{2}\left(\bar{u}_{m}(x, y, z, s ; r)\right)_{\mathrm{xx}}+\left(y^{2}\left(\bar{u}_{m}(x, y, z, s ; r)\right)_{\mathrm{yy}}+\left(z^{2}\left(\bar{u}_{m}(x, y, z, s ; r)\right)_{\mathrm{zz}}\right),\right.\right.
\end{aligned}
$$

and $\tilde{u}_{0}(x, y, z, s ; r)=\tilde{0}$ for $\tilde{0} \in E^{1}$. 
Making the above correction functionals stationary, we have

$$
\begin{aligned}
\delta \underline{u}_{m+1}(x, y, z, t ; r)= & \delta \underline{u}_{m}(x, y, z, t ; r) \\
& +\delta \int_{0}^{t} \lambda\left[\left(\underline{u}_{m}(x, y, z, s ; r)\right)_{s}-(r-1)^{n}(x y z)^{4}-\underline{F}\right] d s, \\
\delta \bar{u}_{m+1}(x, y, z, t ; r)= & \delta \bar{u}_{m}(x, y, z, t ; r) \\
& +\delta \int_{0}^{t} \lambda\left[\left(\bar{u}_{m}(x, y, z, s ; r)\right)_{s}-(1-r)^{n}(x y z)^{4}-\bar{F}\right] d s .
\end{aligned}
$$

Therefore the stationary conditions are defined by

$$
1+\left.\lambda\right|_{s=t}, \quad \lambda^{\prime}(s)=0,
$$

and the Lagrange multiplier is

$$
\lambda=-1
$$

Substituting the value of the Lagrange multiplier into equations (4.56) and (4.57), we obtain the iteration formulas

$$
\begin{aligned}
\underline{u}_{m+1}(x, y, z, t ; r)= & \underline{u}_{m}(x, y, t ; r) \\
& -\int_{0}^{t}\left[\left(\underline{u}_{m}(x, y, z, s ; r)\right)_{s}-(r-1)^{n}(x y z)^{4}-\underline{F}\right] d s, \\
\bar{u}_{m+1}(x, y, z, t ; r)= & \bar{u}_{m}(x, y, s ; r) \\
& -\int_{0}^{t}\left[\left(\bar{u}_{m}(x, y, z, s ; r)\right)_{s}-(1-r)^{n}(x y z)^{4}-\bar{F}\right] d s .
\end{aligned}
$$

Using the fuzzy iteration formulad (4.60) and (4.61) with initial approximation: $\tilde{u}_{0}(x, y$, $t ; r)=\tilde{u}(x, y, t ; r)=\tilde{0}$, we have the following successive approximations:

$$
\left.\begin{array}{rl}
\underline{u}_{1}(x, y, z, t ; r) & =(r-1)^{n} t x^{4} y^{4} z^{4}, \\
\underline{u}_{2}(x, y, z, t ; r) & =(r-1)^{n}\left[t+\frac{t^{2}}{2 !}\right] x^{4} y^{4} z^{4}, \\
\underline{u}_{3}(x, y, z, t ; r) & =(r-1)^{n}\left[t+\frac{t^{2}}{2 !}+\frac{t^{3}}{3 !}\right] x^{4} y^{4} z^{4}, \\
\vdots & \\
\underline{u}_{m}(x, y, z, t ; r) & =(r-1)^{n}\left[t+\frac{t^{2}}{2 !}+\frac{t^{3}}{3 !}+\cdots+\frac{t^{m}}{m !}\right] x^{4} y^{4} z^{4},
\end{array}\right\}
$$


and

$$
\left.\begin{array}{rl}
\bar{u}_{1}(x, y, z, t ; r) & =(1-r)^{n} t x^{4} y^{4} z^{4}, \\
\bar{u}_{2}(x, y, z, t ; r) & =(1-r)^{n}\left[t+\frac{t^{2}}{2 !}\right] x^{4} y^{4} z^{4}, \\
\bar{u}_{3}(x, y, z, t ; r) & =(1-r)^{n}\left[t+\frac{t^{2}}{2 !}+\frac{t^{3}}{3 !}\right] x^{4} y^{4} z^{4}, \\
\vdots & \\
\bar{u}_{m}(x, y, z, t ; r) & =(1-r)^{n}\left[t+\frac{t^{2}}{2 !}+\frac{t^{3}}{3 !}+\cdots+\frac{t^{m}}{m !}\right] x^{4} y^{4} z^{4} .
\end{array}\right\}
$$

Thereafter the exact solution is given by

$$
\begin{aligned}
& \underline{u}(x, y, z, t ; r)=(r-1)^{n}\left[\lim _{m \rightarrow \infty}\left[t+\frac{t^{2}}{2 !}+\frac{t^{3}}{3 !}+\cdots+\frac{t^{m}}{m !}\right] x^{4} y^{4} z^{4}\right], \\
& \bar{u}(x, y, z, t ; r)=(1-r)^{n}\left[\lim _{m \rightarrow \infty}\left[t+\frac{t^{2}}{2 !}+\frac{t^{3}}{3 !}+\cdots+\frac{t^{m}}{m !}\right] x^{4} y^{4} z^{4}\right],
\end{aligned}
$$

and we can rewrite the solution as follows:

$$
\tilde{u}(x, y, z, t ; r)=\left[(r-1)^{n},(1-r)^{n}\right] \odot\left(x^{4} y^{4} z^{4}(\exp (t)-1)\right), \quad 0 \leq r \leq 1 .
$$

Example 4.4 We consider the following one-dimensional fuzzy wave-like equation with variable coefficients:

$$
\tilde{u}_{t t}=\frac{1}{2} x^{2} \odot \tilde{u}_{\mathrm{xx}}, \quad 0<x<1, t>0,
$$

with the boundary conditions

$$
\tilde{u}(0, t)=\tilde{0}, \quad \tilde{u}(1, t)=1+\sinh t,
$$

and the initial conditions

$$
\tilde{u}(x, 0)=\left[r^{n},(2-r)^{n}\right] \odot x, \quad \tilde{u}_{t}(x, 0)=\left[r^{n},(2-r)^{n}\right] \odot x^{2},
$$

where $n=1,2,3, \ldots$.

The parametric form of (4.64) is

$$
\begin{aligned}
& \underline{u}_{t t}=\frac{1}{2} x^{2} \underline{u}_{\mathrm{xx}}, \quad 0<x<1, t>0, \\
& \bar{u}_{t t}=\frac{1}{2} x^{2} \bar{u}_{\mathrm{xx}}, \quad 0<x<1, t>0,
\end{aligned}
$$

for $r \in[0,1]$, where $\underline{u}$ stands for $\underline{u}(x, t ; r)$, and similar for $\bar{u}$.

\section{Case [A]. Fuzzy Adomian decomposition method}

Applying the fuzzy ADM to equations (4.67) and (4.68) yields

$$
L_{t} \underline{u}=\frac{1}{2} x^{2} \underline{u}_{\mathrm{xx}}, \quad 0<x<1,
$$




$$
L_{t} \bar{u}=\frac{1}{2} x^{2} \bar{u}_{\mathrm{xx}}, \quad 0<x<1
$$

where $L_{t}$ and $L_{t}^{-1}$ are the second differential operator and its inverse, respectively. We have the twofold integral operator

$$
L_{t}^{-1}(\cdot)=\int_{0}^{t} \int_{0}^{t}(\cdot) d s d s .
$$

Using equations (4.69) and (4.70) and initial conditions (4.66), we get

$$
\begin{aligned}
& \underline{u}(x, t ; r)=r^{n}\left(x+x^{2} t\right)+\frac{1}{2} L_{t}^{-1}\left(x^{2} \underline{u}_{\mathrm{xx}}\right), \\
& \bar{u}(x, t ; r)=(2-r)^{n}\left(x+x^{2} t\right)+\frac{1}{2} L_{t}^{-1}\left(x^{2} \bar{u}_{\mathrm{xx}}\right) .
\end{aligned}
$$

Using the fuzzy ADM series (3.40) and (3.41) for $\tilde{u}(x, t ; r)=[\underline{u}(x, t ; r), \bar{u}(x, t ; r)]$ in $(4.72)$ and (4.73), we obtain

$$
\begin{aligned}
& \sum_{m=0}^{\infty} \underline{u}_{m}(x, t ; r)=r^{n}\left(x+x^{2} t\right)+\frac{1}{2} L_{t}^{-1}\left[x^{2}\left(\sum_{m=0}^{\infty} \underline{u}_{m}(x, t ; r)\right)_{\mathrm{xx}}\right] \\
& \sum_{m=0}^{\infty} \bar{u}_{m}(x, t ; r)=(2-r)^{n}\left(x+x^{2} t\right)+\frac{1}{2} L_{t}^{-1}\left[x^{2}\left(\sum_{m=0}^{\infty} \bar{u}_{m}(x, t ; r)\right)_{\mathrm{xx}}\right] .
\end{aligned}
$$

Next, use the recursive relations

$$
\left.\begin{array}{l}
\underline{u}_{0}(x, t ; r)=r^{n}\left(x+x^{2} t\right), \\
\underline{u}_{m+1}(x, t ; r)=\frac{1}{2} L_{t}^{-1}\left(x^{2}\left(\underline{u}_{m}\right)_{\mathrm{xx}}\right), \quad m \geq 0,
\end{array}\right\}
$$

and

$$
\left.\begin{array}{l}
\bar{u}_{0}(x, t ; r)=(2-r)^{n}\left(x+x^{2} t\right), \\
\bar{u}_{m+1}(x, t ; r)=\frac{1}{2} L_{t}^{-1}\left(x^{2}\left(\bar{u}_{m}\right)_{\mathrm{xx}}\right), \quad m \geq 0 .
\end{array}\right\}
$$

We have the first few components

$$
\begin{aligned}
& \underline{u}_{0}(x, t ; r)=r^{n}\left(x+x^{2} t\right), \\
& \underline{u}_{1}(x, t ; r)=\frac{1}{2} L_{t}^{-1}\left[x^{2}\left(\underline{u}_{0}\right)_{\mathrm{xx}}\right]=r^{n} x^{2} \frac{t^{3}}{3 !} \\
& \underline{u}_{2}(x, t ; r)=\frac{1}{2} L_{t}^{-1}\left[x^{2}\left(\underline{u}_{1}\right)_{\mathrm{xx}}\right]=r^{n} x^{2} \frac{t^{5}}{5 !}, \\
& \underline{u}_{3}(x, t ; r)=\frac{1}{2} L_{t}^{-1}\left[x^{2}\left(\underline{u}_{2}\right)_{\mathrm{xx}}\right]=r^{n} x^{2} \frac{t^{7}}{7 !},
\end{aligned}
$$


and

$$
\left.\begin{array}{l}
\bar{u}_{0}(x, t ; r)=(2-r)^{n}\left(x+x^{2} t\right), \\
\bar{u}_{1}(x, t ; r)=\frac{1}{2} L_{t}^{-1}\left[x^{2}\left(\bar{u}_{0}\right)_{\mathrm{xx}}\right]=(2-r)^{n} x^{2} \frac{t^{3}}{3 !}, \\
\bar{u}_{2}(x, t ; r)=\frac{1}{2} L_{t}^{-1}\left[x^{2}\left(\bar{u}_{1}\right)_{\mathrm{xx}}\right]=(2-r)^{n} x^{2} \frac{t^{5}}{5 !} \\
\bar{u}_{3}(x, t ; r)=\frac{1}{2} L_{t}^{-1}\left[x^{2}\left(\bar{u}_{2}\right)_{\mathrm{xx}}\right]=(2-r)^{n} x^{2} \frac{t^{7}}{7 !}
\end{array}\right\}
$$

The solution in a fuzzy ADM series form is given by

$$
\begin{aligned}
& \underline{u}(x, t ; r)=r^{n}\left[x+x^{2}\left(t+\frac{t^{3}}{3 !}+\frac{t^{5}}{5 !}+\frac{t^{7}}{7 !}+\cdots\right)\right], \\
& \bar{u}(x, t ; r)=(2-r)^{n}\left[x+x^{2}\left(t+\frac{t^{3}}{3 !}+\frac{t^{5}}{5 !}+\frac{t^{7}}{7 !}+\cdots\right)\right],
\end{aligned}
$$

and thus the exact solution is

$$
\tilde{u}(x, t ; r)=\left[r^{n},(2-r)^{n}\right] \odot\left(x+x^{2} \sinh t\right), \quad 0 \leq r \leq 1 .
$$

\section{Case [B]. Fuzzy variational iteration method}

The correction functionals for equations (4.67) and (4.68) are

$$
\begin{aligned}
& \underline{u}_{m+1}(x, t ; r)=\underline{u}_{m}(x, t ; r)+\int_{0}^{t} \lambda\left[\left(\underline{u}_{m}(x, s ; r)\right)_{s s}-\frac{x^{2}}{2}\left(\underline{u}_{m}(x, s ; r)\right)_{\mathrm{xx}}\right] d s, \\
& \bar{u}_{m+1}(x, t ; r)=\bar{u}_{m}(x, t ; r)+\int_{0}^{t} \lambda\left[\left(\bar{u}_{m}(x, s ; r)\right)_{s s}-\frac{x^{2}}{2}\left(\bar{u}_{m}(x, s ; r)\right)_{\mathrm{xx}}\right] d s,
\end{aligned}
$$

for $m \geq 0$, and $\tilde{u}_{0}(x, t ; r)=\left[r^{n},(2-r)^{n}\right] \odot\left(x+t x^{2}\right)$, where $n=1,2,3, \ldots$.

Making the above correction functional stationary, we have

$$
\begin{aligned}
& \delta \underline{u}_{m+1}(x, t ; r)=\delta \underline{u}_{m}(x, t ; r)+\delta \int_{0}^{t} \lambda\left[\left(\underline{u}_{m}(x, s ; r)\right)_{s s}-\frac{x^{2}}{2}\left(\underline{u}_{m}(x, s ; r)\right)_{\mathrm{xx}}\right] d s, \\
& \delta \bar{u}_{m+1}(x, t ; r)=\delta \bar{u}_{m}(x, t ; r)+\delta \int_{0}^{t} \lambda\left[\left(\bar{u}_{m}(x, s ; r)\right)_{s s}-\frac{x^{2}}{2}\left(\bar{u}_{m}(x, s ; r)\right)_{\mathrm{xx}}\right] d s .
\end{aligned}
$$

Therefore the stationary conditions are

$$
\left.\lambda\right|_{s=t}=0, \quad 1-\left.\lambda^{\prime}\right|_{s=t}=0, \quad \lambda^{\prime \prime}(s)=0 .
$$

By integrating the differential equation we have

$$
\lambda^{\prime \prime}(s)=0 \quad \Rightarrow \quad \lambda(s)=c_{1} s+c_{2} .
$$


Using the boundary conditions, we arrive at

$$
\begin{aligned}
& 1-\left.\lambda^{\prime}\right|_{s=t}=0 \quad \Rightarrow \quad c_{1}=1, \\
& \left.\lambda\right|_{s=t}=0 \quad \Rightarrow \quad c_{2}=-t .
\end{aligned}
$$

Therefore the Lagrange multiplier is

$$
\lambda=s-t
$$

Substituting the value of the Lagrange multiplier into functionals for equations (4.78) and (4.79) gives the iterative formulas

$$
\begin{aligned}
& \underline{u}_{m+1}(x, t ; r)=\underline{u}_{m}(x, t ; r)+\int_{0}^{t}(s-t)\left[\left(\underline{u}_{m}(x, s ; r)\right)_{s s}-\frac{x^{2}}{2}\left(\underline{u}_{m}(x, s ; r)\right)_{x x}\right] d s, \\
& \bar{u}_{m+1}(x, t ; r)=\bar{u}_{m}(x, t ; r)+\int_{0}^{t}(s-t)\left[\left(\bar{u}_{m}(x, s ; r)\right)_{s s}-\frac{x^{2}}{2}\left(\bar{u}_{m}(x, s ; r)\right)_{x x}\right] d s .
\end{aligned}
$$

Using the iterative formula of equations (4.82) and (4.83) with the initial approximation: $\tilde{u}_{0}(x, t ; r)=\left[r^{n},(2-r)^{n}\right] \odot\left(x+t x^{2}\right)$, we get the following successive approximations:

$$
\begin{aligned}
& \underline{u}_{1}(x, t ; r)=r^{n}\left[x+\left(t+\frac{t^{3}}{3 !}\right) x^{2}\right], \\
& \underline{u}_{2}(x, t ; r)=r^{n}\left[x+\left(t+\frac{t^{3}}{3 !}+\frac{t^{5}}{5 !}\right) x^{2}\right], \\
& \underline{u}_{3}(x, t ; r)=r^{n}\left[x+\left(t+\frac{t^{3}}{3 !}+\frac{t^{5}}{5 !}+\frac{t^{7}}{7 !}\right) x^{2}\right],
\end{aligned}
$$

and

$$
\left.\begin{array}{l}
\bar{u}_{1}(x, t ; r)=(2-r)^{n}\left[x+\left(t+\frac{t^{3}}{3 !}\right) x^{2}\right] \\
\bar{u}_{2}(x, t ; r)=(2-r)^{n}\left[x+\left(t+\frac{t^{3}}{3 !}+\frac{t^{5}}{5 !}\right) x^{2}\right] \\
\bar{u}_{3}(x, t ; r)=(2-r)^{n}\left[x+\left(t+\frac{t^{3}}{3 !}+\frac{t^{5}}{5 !}+\frac{t^{7}}{7 !}\right) x^{2}\right]
\end{array}\right\}
$$

So the exact solution is given in the closed form as follows:

$$
\begin{aligned}
& \underline{u}(x, t ; r)=r^{n}\left[\lim _{m \rightarrow \infty} x+\left(t+\frac{t^{3}}{3 !}+\frac{t^{5}}{5 !}+\frac{t^{7}}{7 !}+\cdots\right)\right], \\
& \bar{u}(x, t ; r)=(2-r)^{n}\left[\lim _{m \rightarrow \infty} x+\left(t+\frac{t^{3}}{3 !}+\frac{t^{5}}{5 !}+\frac{t^{7}}{7 !}+\cdots\right)\right],
\end{aligned}
$$


and we can rewrite the solution as follows:

$$
\tilde{u}(x, t ; r)=\left[r^{n},(2-r)^{n}\right] \odot\left(x+x^{2} \sinh t\right), \quad 0 \leq r \leq 1 .
$$

Example 4.5 We consider the following two-dimensional fuzzy wave-like equation with variable coefficients:

$$
\tilde{u}_{t t}=\frac{1}{12}\left(x^{2} \odot \tilde{u}_{\mathrm{xx}} \oplus y^{2} \odot \tilde{u}_{\mathrm{yy}}\right), \quad 0<x, y<1, t>0,
$$

with the Neumann boundary conditions

$$
\begin{array}{ll}
\tilde{u}_{\mathrm{x}}(0, y, t)=\tilde{0}, & \tilde{u}_{\mathrm{x}}(1, y, t)=4 \cosh t, \\
\tilde{u}_{\mathrm{y}}(x, 0, t)=\tilde{0}, \quad \tilde{u}_{\mathrm{y}}(x, 1, t)=4 \sinh t,
\end{array}
$$

and the initial conditions

$$
\begin{aligned}
& \tilde{u}(x, y, 0)=\left[(0.2+0.2 r)^{n},(0.6-0.2 r)^{n}\right] \odot x^{4}, \\
& \tilde{u}_{t}(x, y, 0)=\left[(0.2+0.2 r)^{n},(0.6-0.2 r)^{n}\right] \odot y^{4},
\end{aligned}
$$

where $n=1,2,3, \ldots$.

The parametric form of (4.84) is

$$
\begin{array}{ll}
\underline{u}_{t t}=\frac{1}{12}\left(x^{2} \underline{u}_{\mathrm{xx}}+y^{2} \underline{u}_{\mathrm{yy}}\right), & 0<x, y<1, t>0, \\
\bar{u}_{t t}=\frac{1}{12}\left(x^{2} \bar{u}_{\mathrm{xx}}+y^{2} \bar{u}_{\mathrm{yy}}\right), & 0<x, y<1, t>0,
\end{array}
$$

for $r \in[0,1]$, where $\underline{u}$ stands for $\underline{u}(x, y, t ; r)$, and similarly for $\bar{u}$.

\section{Case [A]. Fuzzy Adomian decomposition method}

In an operator form, equations (4.87) and (4.88) are

$$
\begin{aligned}
& L_{t} \underline{u}=\frac{1}{12}\left(x^{2} \underline{u}_{\mathrm{xx}}+y^{2} \underline{u}_{\mathrm{yy}}\right), \\
& L_{t} \bar{u}=\frac{1}{12}\left(x^{2} \bar{u}_{\mathrm{xx}}+y^{2} \bar{u}_{\mathrm{yy}}\right) .
\end{aligned}
$$

Applying the inverse operator $L^{-1}$ to both sides of equations (4.89) and (4.90), we obtain

$$
\begin{aligned}
& \underline{u}(x, y, t ; r)=(0.2+0.2 r)^{n}\left(x^{4}+y^{4} t\right)+\frac{1}{12} L_{t}^{-1}\left(x^{2} \underline{u}_{\mathrm{xx}}+y^{2} \underline{u}_{\mathrm{yy}}\right), \\
& \bar{u}(x, y, t ; r)=(0.6-0.2 r)^{n}\left(x^{4}+y^{4} t\right)+\frac{1}{12} L_{t}^{-1}\left(x^{2} \bar{u}_{\mathrm{xx}}+y^{2} \bar{u}_{\mathrm{yy}}\right) .
\end{aligned}
$$

Using the fuzzy ADM series for $\tilde{u}(x, y, t ; r)=[\underline{u}(x, y, t ; r), \bar{u}(x, y, t ; r)]$ gives

$$
\begin{aligned}
\sum_{m=0}^{\infty} \underline{u}_{m}(x, y, t ; r)= & (0.2+0.2 r)^{n}\left(x^{4}+y^{4} t\right) \\
& +\frac{1}{12} L_{t}^{-1}\left[x^{2}\left(\sum_{m=0}^{\infty} \underline{u}_{m}(x, y, t ; r)\right)_{\mathrm{xx}}+y^{2}\left(\sum_{m=0}^{\infty} \underline{u}_{m}(x, y, t ; r)\right)_{\mathrm{yy}}\right],
\end{aligned}
$$




$$
\begin{aligned}
\sum_{m=0}^{\infty} \bar{u}_{m}(x, y, t ; r)= & (0.6-0.2 r)^{n}\left(x^{4}+y^{4} t\right) \\
& +\frac{1}{12} L_{t}^{-1}\left[x^{2}\left(\sum_{m=0}^{\infty} \bar{u}_{m}(x, y, t ; r)\right)_{\mathrm{xx}}+y^{2}\left(\sum_{m=0}^{\infty} \bar{u}_{m}(x, y, t ; r)\right)_{\mathrm{yy}}\right] .
\end{aligned}
$$

After that, we use the recursive relations

$$
\left.\begin{array}{l}
\underline{u}_{0}(x, y, t ; r)=(0.2+0.2 r)^{n}\left(x^{4}+y^{4} t\right), \\
\underline{u}_{m+1}(x, y, t ; r)=\frac{1}{12} L_{t}^{-1}\left(x^{2}\left(\underline{u}_{m}\right)_{\mathrm{xx}}+y^{2}\left(\underline{u}_{m}\right)_{\mathrm{yy}}\right), \quad m \geq 0,
\end{array}\right\}
$$

and

$$
\left.\begin{array}{l}
\bar{u}_{0}(x, y, t ; r)=(0.6-0.2 r)^{n}\left(x^{4}+y^{4} t\right), \\
\bar{u}_{m+1}(x, y, t ; r)=\frac{1}{12} L_{t}^{-1}\left(x^{2}\left(\bar{u}_{m}\right)_{\mathrm{xx}}+y^{2}\left(\bar{u}_{m}\right)_{\mathrm{yy}}\right), \quad m \geq 0 .
\end{array}\right\}
$$

The first few components of $\tilde{u}(x, y, t ; r)$ are given by

$$
\left.\begin{array}{l}
\underline{u}_{0}(x, y, t ; r)=(0.2+0.2 r)^{n}\left(x^{4}+y^{4} t\right), \\
\underline{u}_{1}(x, y, t ; r)=\frac{1}{12} L_{t}^{-1}\left[x^{2}\left(\underline{u}_{0}\right)_{\mathrm{xx}}+y^{2}\left(\underline{u}_{0}\right)_{\mathrm{yy}}\right]=(0.2+0.2 r)^{n}\left(x^{4} \frac{t^{2}}{2 !}+y^{4} \frac{t^{3}}{3 !}\right) \\
\underline{u}_{2}(x, y, t ; r)=\frac{1}{12} L_{t}^{-1}\left[x^{2}\left(\underline{u}_{1}\right)_{\mathrm{xx}}+y^{2}\left(\underline{u}_{1}\right)_{\mathrm{yy}}\right]=(0.2+0.2 r)^{n}\left(x^{4} \frac{t^{4}}{4 !}+y^{4} \frac{t^{5}}{5 !}\right) \\
\underline{u}_{3}(x, y, t ; r)=\frac{1}{12} L_{t}^{-1}\left[x^{2}\left(\underline{u}_{2}\right)_{\mathrm{xx}}+y^{2}\left(\underline{u}_{2}\right)_{\mathrm{yy}}\right]=(0.2+0.2 r)^{n}\left(x^{4} \frac{t^{6}}{6 !}+y^{4} \frac{t^{7}}{7 !}\right) \\
\underline{u}_{4}(x, y, t ; r)=\frac{1}{12} L_{t}^{-1}\left[x^{2}\left(\underline{u}_{3}\right)_{\mathrm{xx}}+y^{2}\left(\underline{u}_{3}\right)_{\mathrm{yy}}\right]=(0.2+0.2 r)^{n}\left(x^{4} \frac{t^{8}}{8 !}+y^{4} \frac{t^{9}}{9 !}\right)
\end{array}\right\}
$$

and

$$
\begin{aligned}
\bar{u}_{0}(x, y, t ; r) & =(0.6-0.2 r)^{n}\left(x^{4}+y^{4} t\right), \\
\bar{u}_{1}(x, y, t ; r) & =\frac{1}{12} L_{t}^{-1}\left[x^{2}\left(\bar{u}_{0}\right)_{\mathrm{xx}}+y^{2}\left(\bar{u}_{0}\right)_{\mathrm{yy}}\right]=(0.6-0.2 r)^{n}\left(x^{4} \frac{t^{2}}{2 !}+y^{4} \frac{t^{3}}{3 !}\right), \\
\bar{u}_{2}(x, y, t ; r) & =\frac{1}{12} L_{t}^{-1}\left[x^{2}\left(\bar{u}_{1}\right)_{\mathrm{xx}}+y^{2}\left(\bar{u}_{1}\right)_{\mathrm{yy}}\right]=(0.6-0.2 r)^{n}\left(x^{4} \frac{t^{4}}{4 !}+y^{4} \frac{t^{5}}{5 !}\right), \\
\bar{u}_{3}(x, y, t ; r) & =\frac{1}{12} L_{t}^{-1}\left[x^{2}\left(\bar{u}_{2}\right)_{\mathrm{xx}}+y^{2}\left(\bar{u}_{2}\right)_{\mathrm{yy}}\right]=(0.6-0.2 r)^{n}\left(x^{4} \frac{t^{6}}{6 !}+y^{4} \frac{t^{7}}{7 !}\right) \\
\bar{u}_{4}(x, y, t ; r) & =\frac{1}{12} L_{t}^{-1}\left[x^{2}\left(\bar{u}_{3}\right)_{\mathrm{xx}}+y^{2}\left(\bar{u}_{3}\right)_{\mathrm{yy}}\right]=(0.6-0.2 r)^{n}\left(x^{4} \frac{t^{8}}{8 !}+y^{4} \frac{t^{9}}{9 !}\right) \\
\vdots &
\end{aligned}
$$


Therefore the solution in a series form is

$$
\begin{aligned}
& \underline{u}(x, y, t ; r)=(0.2+0.2 r)^{n}\left[x^{4}\left(1+\frac{t^{2}}{2 !}+\frac{t^{4}}{4 !}+\cdots\right)+y^{4}\left(t+\frac{t^{3}}{3 !}+\frac{t^{5}}{5 !}+\cdots\right)\right], \\
& \bar{u}(x, y, t ; r)=(0.6-0.2 r)^{n}\left[x^{4}\left(1+\frac{t^{2}}{2 !}+\frac{t^{4}}{4 !}+\cdots\right)+y^{4}\left(t+\frac{t^{3}}{3 !}+\frac{t^{5}}{5 !}+\cdots\right)\right],
\end{aligned}
$$

and thus the exact solution is

$$
\tilde{u}(x, y, t ; r)=\left[(0.2+0.2 r)^{n},(0.6-0.2 r)^{n}\right] \odot\left(x^{4} \cosh t+y^{4} \sinh t\right), \quad 0 \leq r \leq 1 .
$$

\section{Case [B]. Fuzzy variational iteration method}

The correction functionals for equations (4.87) and (4.88) with the initial conditions (4.86) are

$$
\begin{aligned}
\underline{u}_{m+1}(x, y, t ; r)= & \underline{u}_{m}(x, y, t ; r)+\int_{0}^{t} \lambda\left[\left(\underline{u}_{m}(x, y, s ; r)\right)_{s s}-\frac{x^{2}}{12}\left(\underline{u}_{m}(x, y, s ; r)\right)_{\mathrm{xx}}\right. \\
& \left.-\frac{y^{2}}{12}\left(\underline{u}_{m}(x, y, s ; r)\right)_{\mathrm{yy}}\right] d s, \\
\bar{u}_{m+1}(x, y, t ; r)= & \bar{u}_{m}(x, y, s ; r)+\int_{0}^{t} \lambda\left[\left(\bar{u}_{m}(x, y, s ; r)\right)_{s s}-\frac{x^{2}}{12}\left(\bar{u}_{m}(x, y, s ; r)\right)_{\mathrm{xx}}\right. \\
& \left.-\frac{y^{2}}{12}\left(\bar{u}_{m}(x, y, s ; r)\right)_{\mathrm{yy}}\right] d s .
\end{aligned}
$$

Making the above correction functional stationary, we have

$$
\begin{aligned}
\delta \underline{u}_{m+1}(x, y, t ; r)= & \delta \underline{u}_{m}(x, y, t ; r)+\delta \int_{0}^{t} \lambda\left[\left(\underline{u}_{m}(x, y, s ; r)\right)_{s s}-\frac{x^{2}}{12}\left(\underline{u}_{m}(x, y, s ; r)\right)_{\mathrm{xx}}\right. \\
& \left.-\frac{y^{2}}{12}\left(\underline{u}_{m}(x, y, s ; r)\right)_{\mathrm{yy}}\right] d s, \\
\delta \bar{u}_{m+1}(x, y, t ; r)= & \delta \bar{u}_{m}(x, y, s ; r)+\delta \int_{0}^{t} \lambda\left[\left(\bar{u}_{m}(x, y, s ; r)\right)_{s s}-\frac{x^{2}}{12}\left(\bar{u}_{m}(x, y, s ; r)\right)_{\mathrm{xx}}\right. \\
& \left.-\frac{y^{2}}{12}\left(\bar{u}_{m}(x, y, s ; r)\right)_{\mathrm{yy}}\right] d s .
\end{aligned}
$$

Similarly, the stationary conditions are obtained by

$$
\left.\lambda\right|_{s=t}=0, \quad 1-\left.\lambda^{\prime}\right|_{s=t}=0, \quad \lambda^{\prime \prime}(s)=0,
$$

and we obtain the Lagrange multiplier

$$
\lambda=s-t .
$$


Substituting the value of the Lagrange multiplier into equations (4.93) and (4.94) gives the iteration formulas

$$
\begin{aligned}
\underline{u}_{m+1}(x, y, t ; r)= & \underline{u}_{m}(x, y, t ; r)+\int_{0}^{t}(s-t)\left[\left(\underline{u}_{m}(x, y, s ; r)\right)_{\mathrm{ss}}-\frac{x^{2}}{12}\left(\underline{u}_{m}(x, y, s ; r)\right)_{\mathrm{xx}}\right. \\
& \left.-\frac{y^{2}}{12}\left(\underline{u}_{m}(x, y, s ; r)\right)_{\mathrm{yy}}\right] d s, \\
\bar{u}_{m+1}(x, y, t ; r)= & \bar{u}_{m}(x, y, s ; r)+\int_{0}^{t}(s-t)\left[\left(\bar{u}_{m}(x, y, s ; r)\right)_{\mathrm{ss}}-\frac{x^{2}}{12}\left(\bar{u}_{m}(x, y, s ; r)\right)_{\mathrm{xx}}\right. \\
& \left.-\frac{y^{2}}{12}\left(\bar{u}_{m}(x, y, s ; r)\right)_{\mathrm{yy}}\right] d s .
\end{aligned}
$$

Using the iteration formula of equations (4.97) and (4.98) with the initial approximation $\tilde{u}_{0}(x, y, t ; r)=\tilde{u}(x, y, t ; r)=\left[(0.2+0.2 r)^{n},(0.6-0.2 r)^{n}\right] \odot\left(x^{4}+t y^{4}\right)$, where $n=1,2,3, \ldots$, we get the following successive approximations:

$$
\begin{aligned}
& \underline{u}_{1}(x, y, t ; r)=(0.2+0.2 r)^{n}\left[x^{4}\left(1+\frac{t^{2}}{2 !}\right)+y^{4}\left(t+\frac{t^{3}}{3 !}\right)\right] \\
& \underline{u}_{2}(x, y, t ; r)=(0.2+0.2 r)^{n}\left[x^{4}\left(1+\frac{t^{2}}{2 !}+\frac{t^{4}}{4 !}\right)+y^{4}\left(t+\frac{t^{3}}{3 !}+\frac{t^{5}}{5 !}\right)\right] \\
& \underline{u}_{3}(x, y, t ; r)=(0.2+0.2 r)^{n}\left[x^{4}\left(1+\frac{t^{2}}{2 !}+\frac{t^{4}}{4 !}+\frac{t^{6}}{6 !}\right)+y^{4}\left(t+\frac{t^{3}}{3 !}+\frac{t^{5}}{5 !}+\frac{t^{7}}{7 !}\right)\right],
\end{aligned}
$$

and

$$
\left.\begin{array}{rl}
\bar{u}_{1}(x, y, t ; r) & =(0.6-0.2 r)^{n}\left[x^{4}\left(1+\frac{t^{2}}{2 !}\right)+y^{4}\left(t+\frac{t^{3}}{3 !}\right)\right], \\
\bar{u}_{2}(x, y, t ; r) & =(0.6-0.2 r)^{n}\left[x^{4}\left(1+\frac{t^{2}}{2 !}+\frac{t^{4}}{4 !}\right)+y^{4}\left(t+\frac{t^{3}}{3 !}+\frac{t^{5}}{5 !}\right)\right], \\
\bar{u}_{3}(x, y, t ; r) & =(0.6-0.2 r)^{n}\left[x^{4}\left(1+\frac{t^{2}}{2 !}+\frac{t^{4}}{4 !}+\frac{t^{6}}{6 !}\right)+y^{4}\left(t+\frac{t^{3}}{3 !}+\frac{t^{5}}{5 !}+\frac{t^{7}}{7 !}\right)\right], \\
\vdots &
\end{array}\right\}
$$

Consequently, the exact solution is given by

$$
\begin{aligned}
& \underline{u}(x, y, t ; r)=(0.2+0.2 r)^{n}\left[\lim _{m \rightarrow \infty} x^{4}\left(1+\frac{t^{2}}{2 !}+\frac{t^{4}}{4 !}+\cdots\right)+y^{4}\left(t+\frac{t^{3}}{3 !}+\frac{t^{5}}{5 !}+\cdots\right)\right], \\
& \bar{u}(x, y, t ; r)=(0.6-0.2 r)^{n}\left[\lim _{m \rightarrow \infty} x^{4}\left(1+\frac{t^{2}}{2 !}+\frac{t^{4}}{4 !}+\cdots\right)+y^{4}\left(t+\frac{t^{3}}{3 !}+\frac{t^{5}}{5 !}+\cdots\right)\right],
\end{aligned}
$$

and we can rewrite the solution as follows:

$$
\tilde{u}(x, y, t, r)=\left[(0.2+0.2 r)^{n},(0.6-0.2 r)^{n}\right] \odot\left(x^{4} \cosh t+y^{4} \sinh t\right), \quad 0 \leq r \leq 1 .
$$


Example 4.6 We consider the following three-dimensional fuzzy wave-like equation with variable coefficients:

$$
\begin{aligned}
\tilde{u}_{t t} & =\left(x^{2}+y^{2}+z^{2}\right) \oplus \frac{1}{2}\left[x^{2} \odot \tilde{u}_{\mathrm{xx}} \oplus y^{2} \odot \tilde{u}_{\mathrm{yy}} \oplus z^{2} \odot \tilde{u}_{\mathrm{zz}}\right], \\
& 0<x, y, z<1, t>0
\end{aligned}
$$

with the boundary conditions

$$
\begin{aligned}
& \tilde{u}(0, y, z, t)=y^{2}\left(\mathrm{e}^{t}-1\right)+z^{2}\left(\mathrm{e}^{-t}-1\right), \\
& \tilde{u}(1, y, z, t)=\left(1+y^{2}\right)\left(\mathrm{e}^{t}-1\right)+z^{2}\left(\mathrm{e}^{-t}-1\right), \\
& \tilde{u}(x, 0, z, t)=x^{2}\left(\mathrm{e}^{t}-1\right)+z^{2}\left(\mathrm{e}^{-t}-1\right), \\
& \tilde{u}(x, 1, z, t)=\left(1+x^{2}\right)\left(\mathrm{e}^{t}-1\right)+z^{2}\left(\mathrm{e}^{-t}-1\right), \\
& \tilde{u}(x, y, 0, t)=\left(x^{2}+y^{2}\right)\left(\mathrm{e}^{t}-1\right), \\
& \tilde{u}(x, y, 1, t)=\left(x^{2}+y^{2}\right)\left(\mathrm{e}^{t}-1\right)+\left(\mathrm{e}^{-t}-1\right),
\end{aligned}
$$

and the initial condition

$$
\tilde{u}(x, y, z, 0)=\tilde{0}, \quad \tilde{u}_{t}(x, y, z, 0)=\left[(0.5 r)^{n},(1-0.5 r)^{n}\right] \oplus\left(x^{2}+y^{2}-z^{2}\right)
$$

where $n=1,2,3, \ldots$, and $\tilde{0} \in E^{1}$.

The parametric form of (4.101) is

$$
\begin{array}{ll}
\underline{u}_{t t}=\left(x^{2}+y^{2}+z^{2}\right)+\frac{1}{2}\left[x^{2} \underline{u}_{\mathrm{xx}}+y^{2} \underline{u}_{\mathrm{yy}}+z^{2} \underline{u}_{\mathrm{zz}}\right], & 0<x, y, z<1, t>0, \\
\bar{u}_{t t}=\left(x^{2}+y^{2}+z^{2}\right)+\frac{1}{2}\left[x^{2} \bar{u}_{\mathrm{xx}}+y^{2} \bar{u}_{\mathrm{yy}}+z^{2} \bar{u}_{\mathrm{zz}}\right], & 0<x, y, z<1, t>0,
\end{array}
$$

for $r \in[0,1]$, where $\underline{u}$ stands for $\underline{u}(x, y, z, t ; r)$, and similarly of $\bar{u}$.

\section{Case [A]. Fuzzy Adomian decomposition method}

In an operator form, equations (4.104) and (4.105) are

$$
\begin{aligned}
& L_{t} \underline{\underline{u}}=\left[\left(x^{2}+y^{2}+z^{2}\right)\right]+\frac{1}{2}\left[x^{2} \underline{u}_{\mathrm{xx}}+y^{2} \underline{u}_{\mathrm{yy}}+y^{2} \underline{u}_{\mathrm{zz}}\right](r), \\
& L_{t} \bar{u}=\left[\left(x^{2}+y^{2}+z^{2}\right)\right]+\frac{1}{2}\left[x^{2} \bar{u}_{\mathrm{xx}}+y^{2} \bar{u}_{\mathrm{yy}}+y^{2} \bar{u}_{\mathrm{zz}}\right](r) .
\end{aligned}
$$

Applying $L_{t}^{-1}$ to equations (4.106) and (4.107), we obtain

$$
\begin{aligned}
\underline{u}(x, y, z, t ; r)= & (0.5 r)^{n}+\left[\left(x^{2}+y^{2}\right)\left(t+\frac{t^{2}}{2 !}\right)+z^{2}\left(-t+\frac{t^{2}}{2 !}\right)\right] \\
& +\frac{1}{2} L_{t}^{-1}\left[x^{2} \underline{u}_{\mathrm{xx}}+y^{2} \underline{u}_{\mathrm{yy}}+y^{2} \underline{u}_{\mathrm{zz}}\right](r),
\end{aligned}
$$




$$
\begin{aligned}
\bar{u}(x, y, z, t ; r)= & (1-0.5 r)^{n}+\left[\left(x^{2}+y^{2}\right)\left(t+\frac{t^{2}}{2 !}\right)+z^{2}\left(-t+\frac{t^{2}}{2 !}\right)\right] \\
& +\frac{1}{2} L_{t}^{-1}\left[x^{2} \bar{u}_{\mathrm{xx}}+y^{2} \bar{u}_{\mathrm{yy}}+y^{2} \bar{u}_{\mathrm{zz}}\right](r) .
\end{aligned}
$$

Using the fuzzy ADM series (3.40) and (3.41) for $\tilde{u}(x, y, z, t ; r)=[\underline{u}(x, y, z, t ; r), \bar{u}(x, y, z, t ; r)]$, we obtain

$$
\begin{aligned}
\sum_{m=0}^{\infty} \underline{u}_{m}(x, y, z, t ; r)= & (0.5 r)^{m}+\left[\left(x^{2}+y^{2}\right)\left(t+\frac{t^{2}}{2 !}\right)+z^{2}\left(-t+\frac{t^{2}}{2 !}\right)\right] \\
& +\frac{1}{2} L_{t}^{-1}\left[x^{2}\left(\sum_{m=0}^{\infty} \underline{u}_{m}(x, y, z, t ; r)\right)_{\mathrm{xx}}\right. \\
& \left.+y^{2}\left(\sum_{m=0}^{\infty} \underline{u}_{m}(x, y, z, t ; r)\right)_{\mathrm{yy}}+z^{2}\left(\sum_{m=0}^{\infty} \underline{u}_{m}(x, y, z, t ; r)\right)_{\mathrm{zz}}\right] \\
\sum_{m=0}^{\infty} \bar{u}_{m}(x, y, z, t ; r)= & (1-0.5 r)^{m}+\left[\left(x^{2}+y^{2}\right)\left(t+\frac{t^{2}}{2 !}\right)+z^{2}\left(-t+\frac{t^{2}}{2 !}\right)\right] \\
& +\frac{1}{2} L_{t}^{-1}\left[x^{2}\left(\sum_{m=0}^{\infty} \bar{u}_{m}(x, y, z, t ; r)\right)_{\mathrm{xx}}\right] \\
& \left.+y^{2}\left(\sum_{m=0}^{\infty} \bar{u}_{m}(x, y, z, t ; r)\right)_{\mathrm{yy}}+z^{2}\left(\sum_{m=0}^{\infty} \bar{u}_{m}(x, y, z, t ; r)\right)_{\mathrm{zz}}\right]
\end{aligned}
$$

Subsequently, we use the recursive relations

$$
\begin{aligned}
& \underline{u}_{0}(x, y, z, t ; r)=(0.5 r)^{n}+\left[\left(x^{2}+y^{2}\right)\left(t+\frac{t^{2}}{2 !}\right)+z^{2}\left(-t+\frac{t^{2}}{2 !}\right)\right], \\
& \underline{u}_{m+1}(x, y, z, t ; r)= \frac{1}{2} L_{t}^{-1}\left[x^{2}\left(\sum_{m=0}^{\infty} \underline{u}_{m}(x, y, t ; r)\right)_{\mathrm{xx}}\right. \\
&\left.+y^{2}\left(\sum_{m=0}^{\infty} \underline{u}_{m}(x, y, t ; r)\right)_{\mathrm{yy}}+z^{2}\left(\sum_{m=0}^{\infty} \underline{u}_{m}(x, y, t ; r)\right)_{\mathrm{zz}}\right], m \geq 0,
\end{aligned}
$$

and

$$
\begin{aligned}
\bar{u}_{0}(x, y, z, t ; r)=(1 & -0.5 r)^{n}+\left[\left(x^{2}+y^{2}\right)\left(t+\frac{t^{2}}{2 !}\right)+z^{2}\left(-t+\frac{t^{2}}{2 !}\right)\right], \\
\bar{u}_{m+1}(x, y, z, t ; r)= & \frac{1}{2} L_{t}^{-1}\left[x^{2}\left(\sum_{m=0}^{\infty} \bar{u}_{m}(x, y, t ; r)\right)_{\mathrm{xx}}\right. \\
& \left.+y^{2}\left(\sum_{m=0}^{\infty} \bar{u}_{m}(x, y, t ; r)\right)_{\mathrm{yy}}+z^{2}\left(\sum_{m=0}^{\infty} \bar{u}_{m}(x, y, t ; r)\right)_{\mathrm{zz}}\right], \quad m \geq 0 .
\end{aligned}
$$


The first few components of $\tilde{u}(x, y, z, t ; r)$ are

$$
\begin{aligned}
\underline{u}_{0}(x, y, z, t ; r) & =(0.5 r)^{n}+\left[\left(x^{2}+y^{2}\right)\left(t+\frac{t^{2}}{2 !}\right)+z^{2}\left(-t+\frac{t^{2}}{2 !}\right)\right], \\
\underline{u}_{1}(x, y, z, t ; r) & =\frac{1}{2} L_{t}^{-1}\left[x^{2}\left(\underline{u}_{0}\right)_{\mathrm{xx}}+y^{2}\left(\underline{u}_{0}\right)_{\mathrm{yy}}+z^{2}\left(\underline{u}_{0}\right)_{\mathrm{zz}}\right] \\
& =(0.5 r)^{n}+\left[\left(x^{2}+y^{2}\right)\left(\frac{t^{3}}{3 !}+\frac{t^{4}}{4 !}\right)+z^{2}\left(-\frac{t^{3}}{3 !}+\frac{t^{4}}{4 !}\right)\right], \\
\underline{u}_{2}(x, y, z, t ; r) & =\frac{1}{2} L_{t}^{-1}\left[x^{2}\left(\underline{u}_{1}\right)_{\mathrm{xx}}+y^{2}\left(\underline{u}_{1}\right)_{\mathrm{yy}}+z^{2}\left(\underline{u}_{1}\right)_{\mathrm{zz}}\right] \\
& =(0.5 r)^{n}+\left[\left(x^{2}+y^{2}\right)\left(\frac{t^{5}}{5 !}+\frac{t^{6}}{6 !}\right)+z^{2}\left(-\frac{t^{5}}{5 !}+\frac{t^{6}}{6 !}\right)\right],
\end{aligned}
$$

and

$$
\begin{aligned}
\bar{u}_{0}(x, y, z, t ; r) & =(1-0.5 r)^{n}+\left[\left(x^{2}+y^{2}\right)\left(t+\frac{t^{2}}{2 !}\right)+z^{2}\left(-t+\frac{t^{2}}{2 !}\right)\right], \\
\bar{u}_{1}(x, y, z, t ; r) & =\frac{1}{2} L_{t}^{-1}\left[x^{2}\left(\bar{u}_{0}\right)_{\mathrm{xx}}+y^{2}\left(\bar{u}_{0}\right)_{\mathrm{yy}}+z^{2}\left(\bar{u}_{0}\right)_{\mathrm{zz}}\right] \\
& =(1-0.5 r)^{n}+\left[\left(x^{2}+y^{2}\right)\left(\frac{t^{3}}{3 !}+\frac{t^{4}}{4 !}\right)+z^{2}\left(-\frac{t^{3}}{3 !}+\frac{t^{4}}{4 !}\right)\right], \\
\bar{u}_{2}(x, y, z, t ; r) & =\frac{1}{2} L_{t}^{-1}\left[x^{2}\left(\bar{u}_{1}\right)_{\mathrm{xx}}+y^{2}\left(\bar{u}_{1}\right)_{\mathrm{yy}}+z^{2}\left(\bar{u}_{1}\right)_{\mathrm{zz}}\right] \\
& =(1-0.5 r)^{n}+\left[\left(x^{2}+y^{2}\right)\left(\frac{t^{5}}{5 !}+\frac{t^{6}}{6 !}\right)+z^{2}\left(-\frac{t^{5}}{5 !}+\frac{t^{6}}{6 !}\right)\right], \\
\vdots &
\end{aligned}
$$

The solution for the fuzzy wave-like equations (4.104) and (4.105) in a series form is given by

$$
\begin{aligned}
\underline{u}(x, y, z, t ; r)= & (0.5 r)^{n}+\left[\left(x^{2}+y^{2}\right)\left(t+\frac{t^{2}}{2 !}+\frac{t^{3}}{3 !}+\frac{t^{4}}{4 !}+\cdots\right)\right. \\
& \left.+z^{2}\left(-t+\frac{t^{2}}{2 !}-\frac{t^{3}}{3 !}+\frac{t^{4}}{4 !}-\cdots\right)\right], \\
\bar{u}(x, y, z, t ; r)= & (1-0.5 r)^{n}+\left[\left(x^{2}+y^{2}\right)\left(t+\frac{t^{2}}{2 !}+\frac{t^{3}}{3 !}+\frac{t^{4}}{4 !}+\cdots\right)\right. \\
& \left.+z^{2}\left(-t+\frac{t^{2}}{2 !}-\frac{t^{3}}{3 !}+\frac{t^{4}}{4 !}-\cdots\right)\right] .
\end{aligned}
$$

The exact solution of equations (4.104) and (4.105) when $\alpha=1$ is

$$
\begin{aligned}
& \tilde{u}(x, y, z, t ; r)=\left[(0.5 r)^{n},(1-0.5 r)^{n}\right] \oplus\left(-\left(x^{2}+y^{2}+z^{2}\right)+\left(x^{2}+y^{2}\right) \mathrm{e}^{t}+z^{2} \mathrm{e}^{-t}\right), \\
& \quad 0 \leq r \leq 1 .
\end{aligned}
$$




\section{Case [B]. Fuzzy variational iteration method}

The correction functionals for equations (4.104) and (4.105) are

$$
\begin{aligned}
\underline{u}_{m+1}(x, y, z, t ; r)= & \underline{u}_{m}(x, y, z, t ; r)+\int_{0}^{t} \lambda\left[\left(\underline{u}_{m}(x, y, z, s ; r)\right)_{\mathrm{ss}}\right. \\
& \left.-\left(x^{2}+y^{2}+z^{2}\right)+(0.5 r)^{n}-\underline{F}\right] d s, \\
\bar{u}_{m+1}(x, y, z, t ; r)= & \bar{u}_{m}(x, y, z, t ; r)+\int_{0}^{t} \lambda\left[\left(\bar{u}_{m}(x, y, z, s ; r)\right)_{\mathrm{ss}}\right. \\
& \left.-\left(x^{2}+y^{2}+z^{2}\right)+(1-0.5 r)^{n}-\bar{F}\right] d s,
\end{aligned}
$$

for $m \geq 0$ and $\tilde{u}_{0}(x, y, z, t ; r)=\left[(0.5 r)^{n},(1-0.5 r)^{n}\right] \oplus\left(x^{2}+y^{2}-z^{2}\right) t$, where $n=1,2,3, \ldots$ Then

$$
\begin{aligned}
& \underline{F}=\frac{1}{2}\left(x^{2}\left(\underline{u}_{m}(x, y, z, s ; r)\right)_{\mathrm{xx}}+\left(y^{2}\left(\underline{u}_{m}(x, y, z, s ; r)\right)_{\mathrm{yy}}+\left(z^{2}\left(\underline{u}_{m}(x, y, z, s ; r)\right)_{\mathrm{zz}}\right),\right.\right. \\
& \bar{F}=\frac{1}{2}\left(x^{2}\left(\bar{u}_{m}(x, y, z, s ; r)\right)_{\mathrm{xx}}+\left(y^{2}\left(\bar{u}_{m}(x, y, z, s ; r)\right)_{\mathrm{yy}}+\left(z^{2}\left(\bar{u}_{m}(x, y, z, s ; r)\right)_{\mathrm{zz}}\right) .\right.\right.
\end{aligned}
$$

Making the above correction functionals stationary, we have

$$
\begin{aligned}
\delta \underline{u}_{m+1}(x, y, z, t ; r)= & \delta \underline{u}_{m}(x, y, z, t ; r)+\delta \int_{0}^{t} \lambda\left[\left(\underline{u}_{m}(x, y, z, s ; r)\right)_{\mathrm{ss}}\right. \\
& \left.-\left(x^{2}+y^{2}+z^{2}\right)+(0.5 r)^{n}-\underline{F}\right] d s, \\
\delta \bar{u}_{m+1}(x, y, z, t ; r)= & \delta \bar{u}_{m}(x, y, z, t ; r)+\delta \int_{0}^{t} \lambda\left[\left(\bar{u}_{m}(x, y, z, s ; r)\right)_{\mathrm{ss}}\right. \\
& \left.-\left(x^{2}+y^{2}+z^{2}\right)+(1-0.5 r)^{n}-\bar{F}\right] d s .
\end{aligned}
$$

Similarly, the stationary conditions are obtained as

$$
\left.\lambda\right|_{s=t}=0, \quad 1-\left.\lambda^{\prime}\right|_{s=t}=0, \quad \lambda^{\prime \prime}(s)=0,
$$

and therefore the Lagrange multiplier is

$$
\lambda=s-t .
$$

Substituting the value of the Lagrange multiplier into functionals (4.112) and (4.113), we obtain the iteration formulas

$$
\begin{aligned}
\underline{u}_{m+1}(x, y, z, t ; r)= & \underline{u}_{m}(x, y, t ; r)+\int_{0}^{t}(s-t)\left[\left(\underline{u}_{m}(x, y, z, s ; r)\right)_{\mathrm{ss}}\right. \\
& \left.-\left(x^{2}+y^{2}+z^{2}\right)+(0.5 r)^{n}-\underline{F}\right] d s, \\
\bar{u}_{m+1}(x, y, z, t ; r)= & \bar{u}_{m}(x, y, s ; r)+\int_{0}^{t}(s-t)\left[\left(\bar{u}_{m}(x, y, z, s ; r)\right)_{\mathrm{ss}}\right. \\
& \left.-\left(x^{2}+y^{2}+z^{2}\right)+(1-0.5 r)^{n}-\bar{F}\right] d s .
\end{aligned}
$$


Therefore the exact solution is the closed form given by

$$
\begin{aligned}
\underline{u}(x, y, z, t ; r)= & (0.5 r)^{n}+\left[\lim _{m \rightarrow \infty}\left[t+\frac{t^{2}}{2 !}+\frac{t^{3}}{3 !}+\cdots\right]\left(x^{2}+y^{2}\right)\right. \\
& \left.+z^{2}\left[-t+\frac{t^{2}}{2 !}-\frac{t^{3}}{3 !}+\cdots\right]\right], \\
\bar{u}(x, y, z, t ; r)= & (1-0.5 r)^{n}+\left[\lim _{m \rightarrow \infty}\left[t+\frac{t^{2}}{2 !}+\frac{t^{3}}{3 !}+\cdots\right]\left(x^{2}+y^{2}\right)\right. \\
& \left.+z^{2}\left[-t+\frac{t^{2}}{2 !}-\frac{t^{3}}{3 !}+\cdots\right]\right],
\end{aligned}
$$

and we can rewrite the solution as follows:

$$
\begin{aligned}
& \tilde{u}(x, y, z, t ; r)=\left[(0.5 r)^{n},(1-0.5 r)^{n}\right] \oplus\left(-\left(x^{2}+y^{2}+z^{2}\right)+\left(x^{2}+y^{2}\right) \mathrm{e}^{t}+z^{2} \mathrm{e}^{-t}\right) \\
& \quad 0 \leq r \leq 1 .
\end{aligned}
$$

Figures 1 and 2 are used to illustrate that the left-hand functions of the $r$-level set of $\tilde{u}$ ( $u$ lower) are always increasing functions of $r$ and the right-hand functions of the $r$-level set of $\tilde{u}$ ( $u$ upper) are always decreasing functions of $r$ in the above examples.

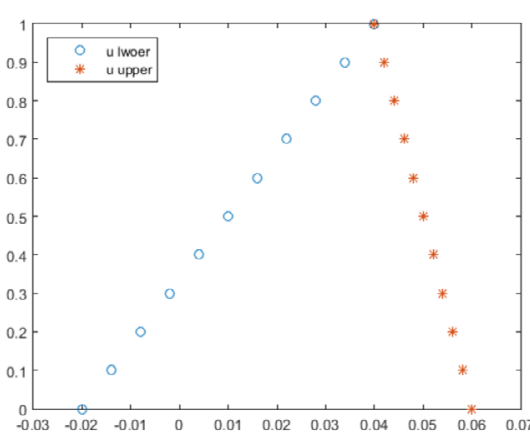

(a)

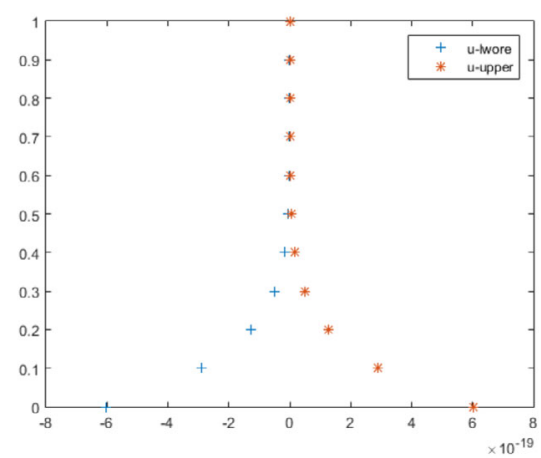

(c)

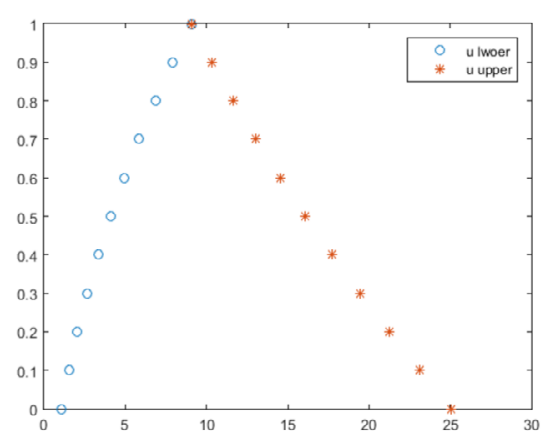

(b)

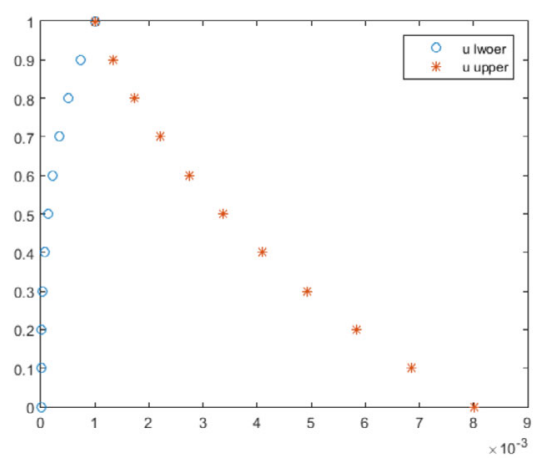

(d)

Figure 1 The results of cases $A$ and $B$ of the first four examples: (a) Example 4.1 when $x=0.2, t=0.001, n=1$; (b) Example 4.2 when $x=0.5, y=0.3, t=0.0001, n=2$; (c) Example 4.3 when $x=0.02, y=0.001, z=0.4, t=5$, $n=7 ;$ (d) Example 4.4 when $x=0.001, t=0.6, n=3$ 


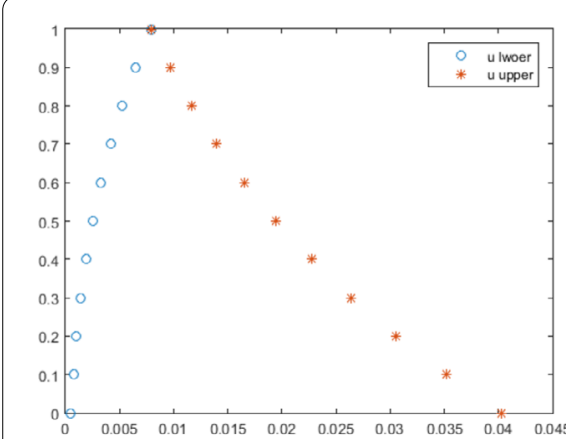

(a)

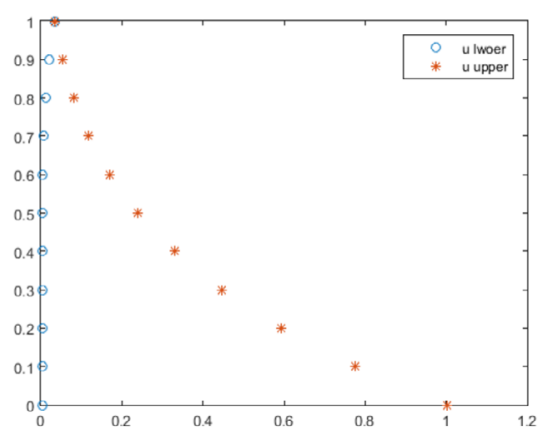

(b)

Figure 2 The results of cases $A$ and $B$ of the second two examples: (a) Example 4.5 when $x=0.04, y=0.8$, $t=0.7, n=4 ;(\mathbf{b})$ Example 4.6 when $x=0.2, y=0.002, z=0.03, t=0.07, n=5$

\section{Conclusions}

In this paper, we investigated the fuzzy Adomian decomposition method (ADM) and fuzzy variational iteration method (VIM) and successfully applied to solving fuzzy heat-like and wave-like equations with variable coefficients under the gH-differentiability. To solve these equations, we first convert each fuzzy partial differential equation with its conditions into two crisp partial differential equations, and then we use ADM and VIM to numerically solve each crisp equation. We illustrate the proposed methods by some numerical examples and use figures to clarify the solutions. The results show that the fuzzy VIM reduces the volume of calculations, whereas tedious calculations are required in the fuzzy ADM; hence the iteration is direct and straightforward. Also, the results reveal that the methods are a very effective, convenient, and accurate mathematical tool for solving fuzzy heat-like and wave-like equations with variable coefficients.

\section{Acknowledgements}

The authors would like to express their sincere appreciation to the editor and anonymous reviewers for their helpful and detailed comments and suggestions, which led to improving the presentation and quality of the work. M. Osman and A.M. Mustafa, we would like to express our gratitude to Prof. Zengtai Gong, not just for introducing us to such an interesting area of research, but also for guiding us through the present work and supporting us during the difficult times we experienced. We have special thanks to the China Scholarship Council (CSC) for a full scholarship at the College of Mathematics and Statistics, Northwest Normal University, Lanzhou, China, in our access to a Ph.D.

Funding

This work is supported by National Natural Science Foundation of China (61763044).

Availability of data and materials

Not applicable.

Competing interests

The authors declare that they have no conflicts of interest.

Authors' contributions

In this paper, all authors contributed equally. The authors read and approved the manuscript.

Author details

${ }^{1}$ College of Mathematics and Statistics, Northwest Normal University, Lanzhou, Gansu, P.R. China. ${ }^{2}$ Department of Applied Mathematics, Faculty of Mathematical Science, University of Khartoum, Khartoum, Sudan.

\section{Publisher's Note}

Springer Nature remains neutral with regard to jurisdictional claims in published maps and institutional affiliations. 


\section{References}

1. Aarayanan, S.L.: An Adomian decomposition method to solve fuzzy Cauchy nonlinear differential equations. Int. J. Sci. Res. Mo. Educ. 2(1), 2455-5630 (2017)

2. Abbasbandy, S., Allahviranloo, T.: Numerical solutions of fuzzy differential equations by Taylor method. J. Comput. Methods Appl. Math. 2, 113-124 (2002)

3. Abbasbandy, S., Allahviranloo, T., Lopez-Pouso, O., Nieto, J.J.: Numerical methods for fuzzy differential inclusions. Comput. Math. Appl. 48, 1633-1641 (2004)

4. Abbod, M.F., Linkens, D.A., Mahfof, M.: Survey of utilisation of fuzzy technology in medicine and healthcare. Fuzzy Sets Syst. 120, 331-349 (2001)

5. Ali, A.H.A., Raslan, K.R.R.: Variational iteration method for solving partial differential equations with variable coefficients. Chaos Solitons Fractals 40, 1520-1529 (2009)

6. Alikhani, R., Bahrami, F.: Fuzzy partial differential equations under the cross product of fuzzy numbers. Inf. Sci. 494 80-99 (2019)

7. Allahviranloo, T.: An analytic approximation to the solution of fuzzy heat equation by Adomian decomposition method. Int. J. Contemp. Math. Sci. 4(3), 105-114 (2008)

8. Allahviranloo, T., Abbasbandy, S., Behzadi, S.S.: Solving nonlinear fuzzy differential equations by using fuzzy variational iteration method. Soft Comput. 18, 2191-2200 (2014)

9. Allahviranloo, T., Ahmady, N., Ahmady, E.: Numerical solution of fuzzy differential equations by predictor-corrector method. Inf. Sci. 177, 1633-1647 (2007)

10. Allahviranloo, T., Gouyandeha, Z., Armanda, A., Hasanoglub, A.: On fuzzy solutions for heat equation based on generalized Hukuhara differentiability. Fuzzy Sets Syst. 265, 1-23 (2015)

11. Bede, B., Gal, S.G.: Generalizations of the differentiability fuzzy-number-valued functions with applications to fuzzy differential equations. Fuzzy Sets Syst. 151, 581-599 (2005)

12. Bede, B., Stefanini, L.: Generalized differentiability of fuzzy-valued functions. Fuzzy Sets Syst. 230, 119-141 (2013)

13. Biswas, S., Roy, T.K.: Adomian decomposition method for fuzzy differential equations with linear differential operator. J. Inf. Comput. Sci. 11(4), 243-250 (2016)

14. Buckley, J.J., Feuring, T.: Fuzzy differential equations. Fuzzy Sets Syst. 110, 43-54 (2000)

15. Cano, Y.C., Flores, H.R.: On new solutions of fuzzy differential equations. Chaos Solitons Fractals 38, 112-119 (2008)

16. Chang, S.L., Zadeh, L.A.: On fuzzy mapping and control. IEEE Trans. Syst. Man Cybern. 2, 30-34 (1972)

17. Datta, D.P.: The golden mean, scale free extension of real number system, fuzzy sets and $\mathrm{i} / \mathrm{f}$ spectrum in physics and biology. Chaos Solitons Fractals 17, 781-788 (2003)

18. Dubois, D., Prade, H.: Toward fuzzy differential calculus: part 3, differentiation. Fuzzy Sets Syst. 8, 225-233 (1982)

19. El Nachie, M.S.: On a fuzzy Kähler manifold which is consistent with slit experiment. Int. J. Nonlinear Sci. Numer. Simul. 6, 95-98 (2005)

20. El Nachie, M.S.: From experimental quantum optics to quantity gravity via a fuzzy Kähler manifold. Chaos Solitons Fractals 25, 969-977 (2005)

21. Fei, W.: Existence and uniqueness of solution for fuzzy random differential equations with non-Lipschitz coefficients. Inf. Sci. 177, 4329-4337 (2007)

22. Friedman, M., Ma, M., Kandel, A.: Numerical solution of fuzzy differential and integral equations. Fuzzy Sets Syst. 106 35-48 (1999)

23. Goetschel, R., Voxman, W.: Elementary fuzzy calculus. Fuzzy Sets Syst. 18, 31-43 (1986)

24. Gong, Z.T., Hao, Y.D.: Fuzzy Laplace transform based on the Henstock integral and its applications in discontinuous fuzzy systems. Fuzzy Sets Syst. 283, 1-28 (2018)

25. Hamoud, A., Azeez, A., Ghadle, K.: A study of some iterative methods for solving fuzzy Volterra-Fredholm integra equations. Indones. J. Electr. Eng. Comput. Sci. 11(3), 1228-1235 (2018)

26. Hamoud, A., Dawood, L., Ghadle, K., Atshan, S.: Usage of the modified variational iteration technique for solving Fredholm integro-differential equations. Int. J. Mech. Prod. Eng. Res. Dev. 9(2), 895-902 (2019)

27. Hamoud, A., Ghadle, K.: Modified Adomian decomposition method for solving fuzzy Volterra-Fredholm integral equations. J. Indian Math. Soc. 85(1-2), 52-69 (2018)

28. Hamoud, A., Ghadle, K.: Modified Laplace decomposition method for fractional Volterra-Fredholm integro-differential equations. J. Math. Model. 6(1), 91-104 (2018)

29. Hamoud, A., Ghadle, K.: The approximate solutions of fractional Volterra-Fredholm integrodifferential equations by using analytical techniques. Probl. Anal. Issues Anal. 7(25), 41-58 (2018)

30. Hamoud, A., Ghadle, K., Atshan, S.: The approximate solutions of fractional integro-differential equations by using modified Adomian decomposition method. Khayyam J. Math. 5(1), 21-39 (2019)

31. He, C.H., He, J.H., Sedighi, H.M.: Fangzhu: an ancient Chinese nanotechnology for water collection from air: history, mathematical insight, promises and challenges. Math. Methods Appl. Sci. (2020). https://doi.org/10.1002/mma.6384

32. He, J.H.: Approximate analytical solution for seepage flow with fractional derivatives in porous media. Comput. Methods Appl. Mech. Eng. 167, 57-68 (1998)

33. He, J.H.: Generalized variational principles for buckling analysis of circular cylinders. Acta Mech. 231, 899-906 (2020)

34. He, J.H.: Variational principle and periodic solution of the Kundu-Mukherjee-Naskar equation. Results Phys. 17, $103031(2020)$

35. He, J.H.: A short review on analytical methods for to a fully fourth-order nonlinear integral boundary value problem with fractal derivatives. Int. J. Numer. Methods Heat Fluid Flow (2020). https://doi.org/10.1108/HFF-01-2020-0060

36. He, J.H., Ain, Q.T.: New promises and future challenges of fractal calculus: from two-scale thermodynamics to fractal variational principle. Therm. Sci. 24(2), Part A, 659-681 (2020)

37. He, J.H., Jin, X.: A short review on analytical methods for the capillary oscillator in a nanoscale deformable tube. Math Methods Appl. Sci. (2020). https://doi.org/10.1002/mma.6321

38. He, J.H., Wu, X.H.: Variational iteration method: new development and applications. Comput. Math. Appl. 54, 881-894 (2007)

39. Jameel, A.F.: Semi-analytical solution of heat equation in fuzzy environment. Int. J. Appl. Phys. Math. 4, 371-378 (2014)

40. Jameel, A.F.: The variational iteration method for solving fuzzy Duffing's equation. J. Interpolat. Approx. Sci. Comput. 2014, Article ID jiasc-00053 (2014) 
41. Jameel, A.F., Anakira, N., Alomari, A.K., Hashim, I., Momani, S.: A new approximation method for solving fuzzy heat equations. J. Comput. Theor. Nanosci. 13, 7825-7832 (2016)

42. Kaleva, O.: Fuzzy differential equations. Fuzzy Sets Syst. 24, 301-317 (1987)

43. Kaleva, O.: The Cauchy problem for fuzzy differential equations. Fuzzy Sets Syst. 35, 389-396 (1990)

44. Lopez, R.R.: Comparison results for fuzzy differential equations. Inf. Sci. 178, 1756-1779 (2008)

45. Ma, M., Friedman, M., Kandel, A.: Numerical solutions of fuzzy differential equations. Fuzzy Sets Syst. 105, 133-138 (1999)

46. Mizukoshi, M.T., Barros, L.C., Chalco-Cano, Y., Romàn-Flores, H., Bassanezi, R.C.: Fuzzy differential equations and the extension principle. Inf. Sci. 177, 3627-3635 (2007)

47. Osman, M., Gong, Z.T., Mohammed, A.: Differential transform method for solving fuzzy fractional wave equation. J. Comput. Anal. Appl. 29(3), 431-453 (2021)

48. Paripour, M., Hajilou, E., Hajilou, A., Heidari, H.: Application of Adomian decomposition method to solve hybrid fuzzy differential equations. J. Taibah Univ. Sci. 9, 95-103 (2015)

49. Pirzada, U.M., Vakaskar, D.C.: Solution of fuzzy heat equations using Adomian decomposition method. Int. J. Adv. Appl. Math. Mech. 3(1), 87-91 (2015)

50. Puri, M.L., Ralescu, D.: Fuzzy random variables. J. Math. Anal. Appl. 114, 409-422 (1986)

51. Puri, M.L., Ralescu, D.A.: Differentials of fuzzy functions. J. Math. Anal. Appl. 91, 552-558 (1983)

52. Shao, Y., Mou, Q., Gong, Z.T.: On retarded fuzzy functional differential equations and nonabsolute fuzzy integrals. Fuzzy Sets Syst. 375, 121-140 (2019)

53. Song, L., Weiguo, W: A new improved Adomian decomposition method and its application to fractional differential equations. Appl. Math. Model. 37(3), 1590-1598 (2013)

54. Stepnicka, M., Valasek, R.: Numerical solution of partial differential equations with help of fuzzy transform. In: The 14th IEEE International Conference on Fuzzy Systems, 2005. FUZZ 05, Reno, NV, USA (2005)

55. Yang, H., Gong, Z: III-posedness for fuzzy Fredholm integral equations of the first kind and regularization methods. Fuzzy Sets Syst. 358, 132-149 (2019)

56. Zhang, H., Liao, X., Yu, J.: Fuzzy modeling and synchronization of hyperchaotic systems. Chaos Solitons Fractals 26 835-843 (2005)

57. Zhang, Y., He, J., Wang, S., Wang, P.: A dye removal model with a fuzzy initial condition. Therm. Sci. 20(3), 867-870 (2016)

58. Zimmermann, H.J.: Fuzzy Set Theory and Its Applications. Kluwer Academic, Dordrecht (1991)

\section{Submit your manuscript to a SpringerOpen ${ }^{\circ}$ journal and benefit from:}

- Convenient online submission

- Rigorous peer review

- Open access: articles freely available online

- High visibility within the field

- Retaining the copyright to your article

Submit your next manuscript at $\gg$ springeropen.com 